\title{
Investigation for Synchronization of a Rotor-Pendulum System considering the Multi-DOF Vibration
}

\author{
Yongjun Hou and Pan Fang \\ School of Mechanical Engineering, Southwest Petroleum University, Chengdu 610500, China \\ Correspondence should be addressed to Pan Fang; ckfangpan@126.com
}

Received 30 May 2015; Accepted 28 September 2015

Academic Editor: Tai Thai

Copyright (C) 2016 Y. Hou and P. Fang. This is an open access article distributed under the Creative Commons Attribution License, which permits unrestricted use, distribution, and reproduction in any medium, provided the original work is properly cited.

\begin{abstract}
This work is a continuation for our published literature for vibration synchronization. A new mechanism, two rotors coupled with a pendulum rod in a multi-DOF vibration system, is proposed to implement coupling synchronization, and the dynamics equation of mechanism is derived by Lagrange equation. In addition, the coupling relationship between the vibrobody and the pendulum rod is ascertained with the Laplace transformation method, based on the dimensionless equation of the dynamics system. The Poincare method is employed to study the synchronization state between the two unbalanced rotors, which is converted into that of existence and the stability of solutions for synchronization-balance equations. The obtained results are supported by computer simulations. It is demonstrated that the values of the spring stiffness coefficient, the length of the pendulum, and the angular installation of the pendulum are important parameters with respect to the synchronous behavior in the rotor-pendulum system.
\end{abstract}

\section{Introduction}

The word "synchronization" is often encountered in both science and daily life. Our surroundings are full of synchronization phenomenon, which is considered as an adjustment of rhythms of oscillating objects due to their internal weak couplings [1]. For example, violinists play in unison, insects in a population emit acoustic or light pulses with a common rate, birds in a flock flap their wings simultaneously, and the heart of a rapidly galloping horse contracts once per locomotory cycle [2]. Synchronization phenomena in large populations of interacting elements are the subjects of intense research efforts in physical, biological, chemical, and social system; however, the most representatives are synchronization of complex systems [3-5], coupled with pendula or mechanical rotors in recent years. For the synchronization of pendula, in the particular case of Huygens' clocks system, the remarkable feature reported by Huygens in 1665 is that pendulum clocks synchronize in antiphase. Nowadays the synchronized limit behavior of Huygens' clocks, synphase and antiphase synchronization of the pendula, is studied considering the different values of spring stiffness [6,7]. Meanwhile, the synchronization of derivatizations of Huygens' clocks, including two coupled double pendula [8], pendulum coupled by an elastic force [9], and pendula connected by linear springs [10], has been attracting many scholars' attention. For the synchronization of rotors, Blekhman [1] proposed the Poincare method for the synchronization state and stability and by now this method is widely used in engineering. Based on Blekhman's method, many scientists have been developing the other methods to analyze the synchronization of the rotors. Wen et al. [11] developed the average method to investigate synchronization and stability of multiple rotors in afterresonance. Zhang et al. [12,13] described the average method of modified small parameters, which immensely simplify the process for solving the problems of synchronization of the rotors. Then, Fang et al. [14] employed the average method of modified small parameters to explore the synchronization of two homodromy rotors installed on a double vibrobody in a coupling vibration system. Sperling et al. [15] presented analytical and numerical investigation of a two-plane automatic balancing device, for equilibration of rigid-rotor unbalance. Balthazar [16, 17] examined self-synchronization of four nonideal exciters in nonlinear vibration system via numerical simulations. Djanan et al. [18] explored the condition, for which three motors working on a same plate can enter into synchronization with the phase difference depending on the physical characteristics of the motors and the plate. 
The abovementioned researches are mainly synchronization of the pendula or the rotors; however, the synchronization of the rotors coupled with pendula is less reported. Recently, we have proposed synchronization of two homodromy rotors coupled with a pendulum rod in an postresonant system [19], but the influence of the multi-DOF vibration of vibrobody on the synchronous state of the rotors is less considered. This paper is a continuation of our published literature by means of the Poincare method, building on the original work of Blekhman. Here, we consider the model of two homodromy rotors coupled with a rigid pendulum rod through a torsion spring, and the vibrobody is connected to a fixed support by means of springs. It is demonstrated that the values of the spring stiffness coefficient, the length of the pendulum, and the angular installation of the pendulum are important parameters with respect to the synchronous behavior in the rotor-pendulum system.

This paper is organized as follows. Section 2 describes the strategy and considered model. In Section 3, we employ the Laplace transform method to calculate the value of the coupling coefficients. In Section 4, we derive the synchronization equation and the synchronization criterion of the system. In Section 5, we compare and analyze the values of the stable phase difference with theoretical computations and the computer simulations. Finally, we summarize our results in Section 6.

\section{Strategy and Model}

2.1. Strategy. Consider the dynamic equation of a rotation system:

$$
\begin{aligned}
J_{s} \ddot{\varphi}_{s}+f_{s} \dot{\varphi}_{s}= & \mu \Phi_{s}\left(\varphi_{s}, \ddot{x}\right) \quad(s=1, \ldots, k), \\
\ddot{x}+2 \omega_{x} \xi_{x} \dot{x}+\omega_{x}^{2} x= & \sum_{j=1}^{k} F_{j}\left(\omega t, \alpha_{1}, \ldots, \alpha_{k}\right) \\
& +\mu F_{k+1}\left(\omega t, \alpha_{1}, \ldots, \alpha_{k}\right),
\end{aligned}
$$

where $\mu \Phi_{s}=M_{e s}+f\left(\varphi_{s}, \ddot{x}\right), \mu$ is a small parameter, $J_{s}$ is the rotational inertia of the sth induction motors, $f_{s}$ is the mechanical damping of the motors, $\xi_{x}$ is the damping ratio of the system in the $x$-direction, and $\omega_{x}$ is natural frequency of the system in $x$-direction. $\omega$ and $\varphi_{s}$ are mechanical velocity and phase angular of the $s$ th unbalanced rotor, respectively.

Based on (1), the following sequence of analysis for vibration system employing synchronizing rotors can be formulated:

(1) Steady forced vibrations with $T=2 \pi / \omega$ are determined by

$$
x=x\left(\omega t, \alpha_{1}, \ldots, \alpha_{k}\right)
$$

from the supporting body or supporting system of bodies (i.e., from the second formula of (1) considering $\mu=0$ ) when rotors are uniformly rotating with initial phase $\alpha_{1}, \ldots, \alpha_{k}$; that is,

$$
\varphi_{s}=\left(\omega t+\alpha_{s}\right) \text {. }
$$

(2) Equation (3) mentioned above may correspond only to such values of constants $\alpha_{1}, \ldots, \alpha_{k}$, which satisfy

$$
P_{s}\left(\alpha_{1}, \ldots, \alpha_{k}\right)=\mu\left\langle\Phi_{s}\left(\varphi_{s}, \ddot{x}\right)\right\rangle=0 .
$$

Here and below the angle brackets $\langle *\rangle$ show the average within $T$; that is,

$$
\langle *\rangle=\frac{1}{T} \int_{0}^{T} * d t,
$$

where symbol $*$ represents a function related to time $t[1]$.

(3) If a certain set of constants $\alpha_{1}=\alpha_{1}^{*}, \ldots, \alpha_{k}=\alpha_{k}^{*}$, which satisfy (4), real parts of all roots $\chi$ of the $k$ th order algebraic equation

$$
\begin{aligned}
& \left|\begin{array}{cccc}
\frac{\partial\left(P_{1}-P_{k}\right)}{\partial \alpha_{1}}-\chi & \frac{\partial\left(P_{1}-P_{k}\right)}{\partial \alpha_{2}} & \cdots & \frac{\partial\left(P_{1}-P_{k}\right)}{\partial \alpha_{k-1}} \\
\frac{\partial\left(P_{2}-P_{k}\right)}{\partial \alpha_{1}} & \frac{\partial\left(P_{2}-P_{k}\right)}{\partial \alpha_{2}}-\chi & \cdots & \frac{\partial\left(P_{2}-P_{k}\right)}{\partial \alpha_{k-1}} \\
\cdots & \cdots & \cdots \\
\frac{\partial\left(P_{k-1}-P_{k}\right)}{\partial \alpha_{1}} & \frac{\partial\left(P_{k-1}-P_{k}\right)}{\partial \alpha_{2}} & \cdots & \frac{\partial\left(P_{k-1}-P_{k}\right)}{\partial \alpha_{k-1}}-\chi
\end{array}\right| \\
& \quad=0,
\end{aligned}
$$

are negative, then at sufficiently small $\mu$ this set of constants is indeed correlated with the unique, analytical relative to $\mu$, asymptotically stale periodic solution of (1). This solution changes into the fundamental solution (see (4)) at $\mu=0$. If the real part of at least one root of (6) is positive, then the corresponding solution is unstable. With purely imaginary zero roots, an additional analysis is required in general case [1].

2.2. Model. A simplified rotor-pendulum system depicted in Figure 1(a) is considered. This model consists of a rigid vibrobody of mass $m_{0}[\mathrm{Kg}]$, a rigid pendulum rod, and two unbalanced rotors. The rigid vibrobody is elastically supported via linear springs with stiffness $k_{x}[\mathrm{~N} / \mathrm{m}], k_{y}[\mathrm{~N} / \mathrm{m}]$, and $k_{\psi}[\mathrm{N} / \mathrm{rad}]$ and linear viscous dampers with damping constants $f_{x}[\mathrm{Ns} / \mathrm{m}], f_{y}[\mathrm{Ns} / \mathrm{m}]$, and $f_{\psi}[\mathrm{Nm} /(\mathrm{rad} / \mathrm{s})]$, respectively. Unbalanced rotor $i$ is modelled by a point mass $m_{i}[\mathrm{Kg}]$ (for $i=2,3$ ) attached at the end of a massless rod of length $r[\mathrm{~m}]$. One of the unbalanced rotors in the system is directly mounted on the vibrobody, and the other is fixed at the end of a pendulum rod, which is connected with the vibro-platform by a linear torque spring with stiffness $k_{1}[\mathrm{~N} / \mathrm{rad}]$ and a linear viscous damper with damping constant $f_{1}[\mathrm{Nm} /(\mathrm{rad} / \mathrm{s})]$. Note that the two rotors are driven separately in the same direction by two identical induction motors. The rotation angle of rotor $i$ is denoted by $\varphi_{i} \in S^{1}$ (for $i=2,3$ ) in [rad]; the oscillating angle of the pendulum rod is denoted by $\varphi_{1} \in S^{1}$ in [rad]; the installation angle of the pendulum rod is expressed by $\beta \in S^{1}$ 


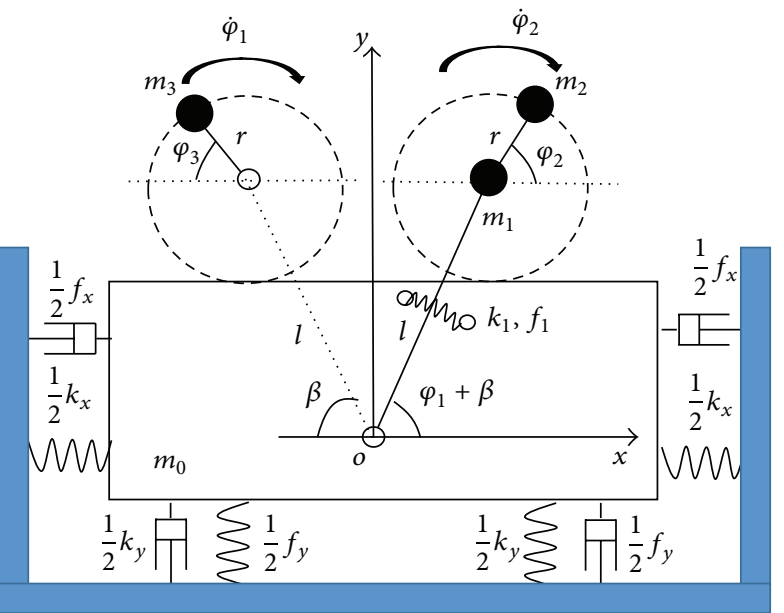

(a)

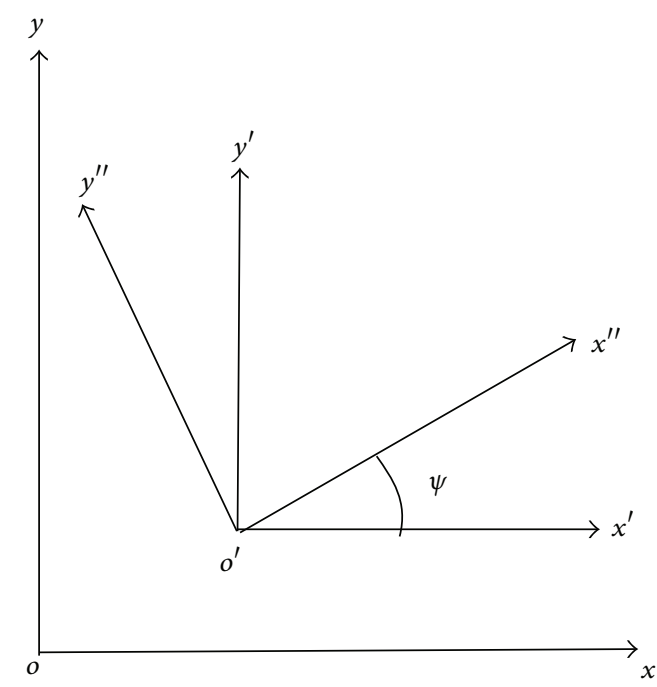

(b)

FIGURE 1: The model of the vibration system: (a) dynamic model of the double vibrobody system with two induction motors rotating in the same direction and (b) the reference frame system.

in $[\mathrm{rad}] ; M_{i}$ represents the electromagnetic torque of the induction motors in $[\mathrm{N} / \mathrm{m}] ; R_{i}$ signifies resistance torque in bearings; and $x, y$, and $\psi$ are the vibration displacement of the vibrobody in $[\mathrm{m}],[\mathrm{m}]$, and $[\mathrm{rad}]$, respectively.

As illustrated in Figure 1(b), three reference coordinates of the system can be assigned as follows: the fixed coordinate $o x y$; the nonrotating moving coordinate $o^{\prime} x^{\prime} y^{\prime}$, which undergoes the translation motion while remaining parallel to coordinate $o x y$, respectively; the rotating coordinate $o^{\prime} x^{\prime \prime} y^{\prime \prime}$ which dedicates the rotation motion around points $o^{\prime}$. The three reference coordinates of the vibrobody separately coincide with each other when the system is in the static equilibrium state.

In the reference frame $o^{\prime} x^{\prime \prime} y^{\prime \prime}$, the center coordinate $\Phi_{i}^{\prime \prime}$ $(i=1,2,3)$ of the rotors and pendula can be expressed as

$$
\begin{aligned}
& \boldsymbol{\Phi}_{1}^{\prime \prime}=\left(\begin{array}{c}
l \cos \left(\beta+\varphi_{1}\right) \\
l \sin \left(\beta+\varphi_{1}\right)
\end{array}\right), \\
& \boldsymbol{\Phi}_{2}^{\prime \prime}=\left(\begin{array}{c}
l \cos \left(\beta+\varphi_{1}\right)+r \cos \varphi_{2} \\
l \sin \left(\beta+\varphi_{1}\right)+r \sin \varphi_{2}
\end{array}\right), \\
& \boldsymbol{\Phi}_{3}^{\prime \prime}=\left(\begin{array}{c}
-l \cos \beta+r \cos \varphi_{3} \\
l \sin \beta+r \sin \varphi_{3}
\end{array}\right) .
\end{aligned}
$$

In the reference frame $o x y$, the center coordinate of the rotors and pendula, $\boldsymbol{\Phi}_{i}(i=1,2,3)$, can be expressed as

$$
\begin{aligned}
\boldsymbol{\Phi}_{i} & =\boldsymbol{\Phi}_{0}+\boldsymbol{\Gamma} \boldsymbol{\Phi}_{i}^{\prime \prime}, \\
\boldsymbol{\Gamma} & =\left(\begin{array}{cc}
\cos \psi & -\sin \psi \\
\sin \psi & \cos \psi
\end{array}\right),
\end{aligned}
$$

where $\Phi_{0}$ is displacement vector of the mass center of the rigid vibrobody, $\boldsymbol{\Phi}_{0}=[x, y]^{T}$.
The kinetic energy of the system can be obtained by

$$
\begin{aligned}
T= & \frac{1}{2} m\left(\dot{x}^{2}+\dot{y}^{2}\right)+\frac{1}{2} J_{m} \dot{\psi}^{2}+\frac{1}{2} \sum_{i=1}^{3} J_{0 i} \dot{\varphi}_{i}^{2} \\
& +\frac{1}{2} \sum_{i=1}^{3} m_{i} \dot{\boldsymbol{\Phi}}_{i}^{T} \dot{\boldsymbol{\Phi}}_{i} .
\end{aligned}
$$

The potential energy of the system is approximated to

$$
V=\frac{1}{2} k_{x} x^{2}+\frac{1}{2} k_{y} y^{2}+\frac{1}{2} k_{\psi} \psi^{2}+\frac{1}{2} k_{1} \varphi_{1}^{2}
$$

Moreover, the viscous dissipation function $D$ of the system is expressed by

$$
\begin{aligned}
D= & \frac{1}{2} f_{x} \dot{x}^{2}+\frac{1}{2} f_{y} \dot{y}^{2}+\frac{1}{2} f_{\psi} \dot{\psi}^{2}+\frac{1}{2} f_{1} \dot{\varphi}_{1}^{2}+\frac{1}{2} f_{2} \dot{\varphi}_{2}^{2} \\
& +\frac{1}{2} f_{3} \dot{\varphi}_{3}^{2} .
\end{aligned}
$$

The dynamics equation is obtained by using Lagrange's equation:

$$
\frac{d}{d t} \frac{\partial T}{\partial \dot{q}_{i}}-\frac{\partial(T-V)}{\partial q_{i}}+\frac{\partial D}{\partial \dot{q}_{i}}=Q_{i}
$$

If $\mathbf{q}=\left[x, y, \psi, \varphi_{1}, \varphi_{2}, \varphi_{3}\right]^{T}$ is chosen as the generalized coordinates, the generalized force are $Q_{x}=Q_{y}=Q_{\psi}=0$, $Q_{\varphi 1}=M_{e 1}-R_{e 1}, Q_{\varphi 2}=T_{e 2}-R_{e 2}$, and $Q_{\varphi 3}=0$, respectively. As $m_{i} \ll m$ and $\psi, \varphi_{3} \ll 1$ in the system, the inertia coupling stemming from asymmetry of the two rotors can be neglected. Substituting (9), (10), and (11) into (12), we can 
yield the dynamic equation of the vibration system as the following form:

$$
\begin{aligned}
& M \ddot{x}+f_{x} \dot{x}+k_{x} x=\left(m_{1}+m_{2}\right) l\left[\dot{\varphi}_{1}^{2} \cos \beta+\ddot{\varphi}_{1} \sin \beta_{1}\right] \\
& +\sum_{i=2}^{3} m_{i} r\left[\ddot{\varphi}_{i} \sin \varphi_{i}+\dot{\varphi}_{i}^{2} \cos \varphi_{i}\right], \\
& M \ddot{y}+f_{y} \dot{y}+k_{y} y=\left(m_{1}+m_{2}\right) l\left[\dot{\varphi}_{1}^{2} \sin \beta-\ddot{\varphi}_{1} \cos \beta\right] \\
& +\sum_{i=2}^{3} m_{i} r\left[\dot{\varphi}_{i}^{2} \sin \varphi_{i}-\ddot{\varphi}_{i} \cos \varphi_{i}\right], \\
& J_{m 0} \ddot{\psi}+f_{\psi} \dot{\psi}+k_{\psi} \psi=\sum_{i=2}^{3}(-1)^{i} \\
& \cdot m_{i} l r\left[\dot{\varphi}_{i}^{2} \sin \left(\varphi_{i}-(-1)^{i} \beta\right)\right. \\
& \left.-\ddot{\varphi}_{2} \cos \left(\varphi_{i}-(-1)^{i} \beta\right)\right], \\
& \left(m_{1} l^{2}+m_{2} l^{2}\right) \ddot{\varphi}_{1}+f_{1} \dot{\varphi}_{1}+k_{1} \varphi_{1}=\left(m_{1}+m_{2}\right) l[\ddot{x} \sin \beta \\
& -\ddot{y} \cos \beta]-m_{2} \operatorname{lr}\left[\ddot{\varphi}_{2} \cos \left(\varphi_{2}-\beta\right)-\dot{\varphi}_{2}^{2}\right. \\
& \left.\cdot \sin \left(\varphi_{2}-\beta\right)\right] \\
& J_{20} \ddot{\varphi}_{2}=M_{e 1}-R_{e 1}-m_{2} r\left[-\ddot{x} \sin \varphi_{2}+\ddot{y} \cos \varphi_{2}\right] \\
& -m_{2} l r\left[\left(\ddot{\varphi}_{1}+\ddot{\psi}\right) \cos \left(\varphi_{2}-\beta\right)+\left(\dot{\varphi}_{1}+\dot{\psi}\right)^{2}\right. \\
& \left.\cdot \sin \left(\varphi_{2}-\beta\right)\right] \\
& J_{30} \ddot{\varphi}_{3}=M_{e 2}-R_{e 2}-m_{3} r\left[-\ddot{x} \sin \varphi_{3}+\ddot{y} \cos \varphi_{3}\right] \\
& +m_{3} \operatorname{lr}\left[\ddot{\psi} \cos \left(\varphi_{3}+\beta\right)+\dot{\psi}^{2} \sin \left(\varphi_{3}+\beta\right)\right],
\end{aligned}
$$

where $\sum_{i=0}^{3} m_{i}=M, J_{m 0} \approx M l_{e}^{2}+m_{1} l^{2}, J_{20} \approx m_{2} r^{2}+m_{3} r^{2}$, and $J_{30} \approx m_{3} r_{3}^{2}$.

Assuming that the natural frequency of the system is unequal to or far away from the excitation frequency, thus the oscillating angle $\varphi_{1}$ of pendulum rod can be considered small and periodic:

$$
\varphi_{1}=A \sin (\omega t+\gamma)
$$

where $A$ is amplitude of the oscillating angle of pendulum rod (here, $A \ll 1$ ) and $\gamma$ is the phase angular of the pendulum rod.

Having calculated the derivative of the function $\varphi_{1}(t)$ with respect to $t$, we will write the relationship under consideration as

$$
\frac{\dot{\varphi}_{1}^{2}}{\ddot{\varphi}_{1}}=\frac{A^{2} \omega^{2} \sin ^{2}(\omega t+\gamma)}{A \omega^{2} \sin (\omega t+\gamma)}=A \sin (\omega t+\gamma) \sim \mu .
$$

Hence, it can be seen that the items related to symbol $\dot{\varphi}_{1}^{2}$ in (13) are nonlinear; however, the nonlinear characteristics and dampers acted on the system are weaker when the values of the parameters in the system satisfy the assumptions above. Meanwhile, considering the solution of the problem by the
Poincare method (i.e., based on the fundamental equation (1)), we will introduce the small parameter $\mu$ into (13), thus presenting it in the following form:

$$
\begin{aligned}
& M \ddot{x}+k_{x} x=\left(m_{1}+m_{2}\right) l \ddot{\varphi}_{1} \sin \beta_{1}+\sum_{i=2}^{3} m_{i} r\left[\ddot{\varphi}_{i} \sin \varphi_{i}\right. \\
& \left.\quad+\dot{\varphi}_{i}^{2} \cos \varphi_{i}\right]-\mu\left[f_{x}^{\prime} \dot{x}-\left(m_{1}+m_{2}\right) l \dot{\varphi}_{1}^{2} \cos \beta\right], \\
& M \ddot{y}+k_{y} y=-\left(m_{1}+m_{2}\right) l \ddot{\varphi}_{1} \cos \beta \\
& \quad+\sum_{i=2}^{3} m_{i} r\left[\dot{\varphi}_{i}^{2} \sin \varphi_{i}-\ddot{\varphi}_{i} \cos \varphi_{i}\right]-\mu\left[f_{y}^{\prime} \dot{y}-\left(m_{1}\right.\right. \\
& \left.\left.\quad+m_{2}\right) l \dot{\varphi}_{1}^{2} \sin \beta\right], \\
& J_{m 0} \ddot{\psi}+k_{\psi} \psi=-m_{1} l^{2} \ddot{\varphi}_{1}+\sum_{i=2}^{3}(-1)^{i} \\
& \quad \cdot m_{i} l r\left[\dot{\varphi}_{i}^{2} \sin \left(\varphi_{i}-(-1)^{i} \beta\right)\right. \\
& \left.\quad-\ddot{\varphi}_{2} \cos \left(\varphi_{i}-(-1)^{i} \beta\right)\right]-\mu f_{\psi}^{\prime} \dot{\psi}, \\
& \left(m_{1} l^{2}+m_{2} l^{2}\right) \ddot{\varphi}_{1}+k_{1} \varphi_{1}=\left(m_{1}+m_{2}\right) l[\ddot{x} \sin \beta-\ddot{y} \\
& \quad \cdot \cos \beta]-m_{2} l r\left[\ddot{\varphi}_{2} \cos \left(\varphi_{2}-\beta\right)-\dot{\varphi}_{2}^{2} \sin \left(\varphi_{2}-\beta\right)\right] \\
& \quad-\mu f_{1}^{\prime} \dot{\varphi}_{1}, \\
& J_{20} \ddot{\varphi}_{2}=\mu \ddot{\varphi}_{1}\left(\varphi_{1}, \ddot{x}\right)
\end{aligned}
$$

where

$$
\begin{aligned}
& \mu \Phi_{1}=M_{e 1}-R_{e 1}-m_{2} r\left[-\ddot{x} \sin \varphi_{2}+\ddot{y} \cos \varphi_{2}\right] \\
& +m_{2} l r\left[\left(\ddot{\varphi}_{1}+\ddot{\psi}\right) \cos \left(\varphi_{2}-\beta\right)\right. \\
& \left.\quad+\left(\dot{\varphi}_{1}+\dot{\psi}\right)^{2} \sin \left(\varphi_{2}-\beta\right)\right], \\
& \mu \Phi_{2}=M_{e 2}-R_{e 2}-m_{3} r\left[-\ddot{x} \sin \varphi_{3}+\ddot{y} \cos \varphi_{3}\right] \\
& \quad-m_{3} l r\left[\ddot{\psi} \cos \left(\varphi_{3}+\beta\right)+\dot{\psi}^{2} \sin \left(\varphi_{3}+\beta\right)\right] .
\end{aligned}
$$

According to [12], when the two rotors synchronously rotate, the electromagnetic torque of the inductions $M_{e i}$ can be linearized at the vicinity of $\dot{\varphi}_{i}=\omega_{s}$ as

$$
M_{e i}=n_{p} \frac{L_{m i}^{2} U_{S 0}^{2}}{L_{s i}^{2} \omega_{m} R_{r i}}\left(\omega_{s}-n_{p} \omega_{m}\right) \text {, }
$$

where $L_{m i}$ is the mutual inductance of the $i$ th induction motor, $L_{s i}$ is stator inductance of the $i$ th induction motor, $n_{p}$ is the number of pole pairs of the induction motor, $\omega_{m}$ is synchronous electric angular velocity, $R_{r i}$ is the rotor resistance of the $i$ th induction motor, and $U_{S 0}$ is the amplitude of the stator voltage vector. 


\section{Steady Response}

The first four formulas in (16) are coupling dynamics equations related to DOFs $x, y, \psi$, and $\varphi_{1}$. When the system operates in the steady state, the acceleration of the three motors changes very little (close to zero), so $\ddot{\varphi}_{2}$ and $\ddot{\varphi}_{3}$ can be neglected in these formulas. In addition, taking no account of the items related to small parameter $\mu$ and introducing the following dimensionless parameters in the mentioned formulas,

$$
\begin{aligned}
& \omega_{x}=\sqrt{\frac{k_{x}}{M}}, \\
& \omega_{y}=\sqrt{\frac{k_{y}}{M}}, \\
& \omega_{\psi}=\sqrt{\frac{k_{\psi}}{\left(M l_{e}^{2}+m_{1} l^{2}\right)}}, \\
& \omega_{1}=\sqrt{\frac{k_{1}}{\left(m_{1} l^{2}+m_{2} l^{2}\right)}}, \\
& r_{0}=\frac{l}{l_{e}}, \\
& r_{l}=\frac{r}{l} \text {, } \\
& r_{m}=\frac{m_{3}}{M} \\
& \eta_{13}=\frac{m_{1}}{m_{3}} \\
& \eta_{23}=\frac{m_{2}}{m_{3}} \\
& f_{1}(t)=\left(\eta_{23} r_{m} r \cos \varphi_{2}+r_{m} r \cos \varphi_{3}\right), \\
& f_{2}(t)=\left(\eta_{23} r_{m} r \sin \varphi_{2}+r_{m} r \sin \varphi_{3}\right) \text {, } \\
& f_{3}(t)=\frac{\eta_{23} r_{m} r r_{o}}{\left(1+\eta_{13} r_{m} r_{o}^{2}\right) l_{e}} \sin \left(\varphi_{2}-\beta\right) \\
& -\frac{r_{m} r r_{o}}{\left(1+\eta_{13} r_{m} r_{o}^{2}\right) l_{e}} \sin \left(\varphi_{3}+\beta\right), \\
& f_{4}(t)=\frac{\eta_{23} r_{l}}{\left(\eta_{13}+\eta_{23}\right)} \sin \left(\varphi_{2}-\beta\right) \text {. }
\end{aligned}
$$

We obtain the dimensionless dynamics equations:

$$
\begin{aligned}
\ddot{x}+\omega_{x}^{2} x= & \left(\eta_{13}+\eta_{23}\right) r_{m} l \ddot{\varphi}_{1} \sin \beta \\
& +\omega^{2} f_{1}(t), \\
\ddot{y}+\omega_{y}^{2} y= & -\left(\eta_{13}+\eta_{23}\right) r_{m} l \ddot{\varphi}_{1} \cos \beta \\
& +\omega^{2} f_{2}(t),
\end{aligned}
$$

$$
\begin{aligned}
\ddot{\psi}+\omega_{\psi}^{2} \psi= & -\frac{\eta_{13} r_{m} r_{o}^{2}}{\left(1+\eta_{13} r_{m} r_{o}^{2}\right)} \ddot{\varphi}_{1} \\
& +\omega^{2} f_{3}(t), \\
\ddot{\varphi}_{1}+2 \omega_{1} \xi_{1} \dot{\varphi}_{1}+\omega_{1}^{2} \varphi_{1}= & \frac{\ddot{x} \sin \beta-\ddot{y} \cos \beta}{l}+\omega^{2} f_{4}(t) .
\end{aligned}
$$

It can be seen that (20) are coupling dynamic equations related to DOFs $x, y, \psi$, and $\varphi_{1}$. Applying the Laplace transform to (20), one gets

$$
\begin{aligned}
s^{2} X(s)+\omega_{x}^{2} X(s)= & \left(\eta_{13}+\eta_{23}\right) r_{m} l s^{2} \sin \beta \Phi(s) \\
& +\omega^{2} F_{1}(s), \\
s^{2} Y(s)+\omega_{y}^{2} Y(s)=- & \left(\eta_{13}+\eta_{23}\right) r_{m} l s^{2} \cos \beta \Phi(s) \\
& +\omega^{2} F_{2}(s), \\
s^{2} \Psi(s)+\omega_{\psi}^{2} \Psi(s)= & -\frac{\eta_{13} r_{m} r_{o}^{2}}{\left(1+\eta_{13} r_{m} r_{o}^{2}\right)} s^{2} \Phi(s) \\
& +\omega^{2} F_{3}(s), \\
& s^{2}{\sin \beta X(s)-s^{2} \cos \beta Y(s)}_{l}^{l} \\
& +\omega^{2} F_{4}(s) .
\end{aligned}
$$

Considering that the natural frequencies of the springs connected to the foundation are approximately identical (i.e., $\left.\omega_{x} \approx \omega_{y} \approx \omega_{\psi}\right)$, thus, $X(s), Y(s), \Psi(s)$, and $\Phi(s)$ can be expressed:

$$
\begin{aligned}
& X(s) \\
& =\left[\frac{\left(\eta_{13}+\eta_{23}\right) r_{m} l \omega^{2} s^{4} \sin ^{2} \beta}{G_{1}(s)}+\frac{\omega^{2}}{\left(s^{2}+\omega_{x}^{2}\right)}\right] F_{1}(s) \\
& \quad-\frac{\left(\eta_{13}+\eta_{23}\right) r_{m} l s^{4} \omega^{2} \sin \beta \cos \beta}{G_{1}(s)} F_{2}(s) \\
& +\frac{\left(\eta_{13}+\eta_{23}\right) r_{m} l s^{2} \omega^{2} \sin \beta}{G_{2}(s)} F_{4}(s),
\end{aligned}
$$

$Y(s)$

$$
\begin{aligned}
& =\left[\frac{-\left(\eta_{13}+\eta_{23}\right) r_{m} l s^{4} \omega^{2} \cos \beta \sin \beta}{G_{1}(s)}\right] F_{1}(s) \\
& +\left[\frac{\left(\eta_{13}+\eta_{23}\right) r_{m} l s^{4} \omega^{2} \cos ^{2} \beta}{G_{1}(s)}+\frac{\omega^{2}}{\left(s^{2}+\omega_{y}^{2}\right)}\right] F_{2}(s) \\
& +\frac{\left(\eta_{13}+\eta_{23}\right) r_{m} l s^{2} \omega^{2} \cos \beta}{G_{2}(s)} F_{4}(s),
\end{aligned}
$$


$\Psi(s)$

$$
\begin{aligned}
= & -\frac{\eta_{13} r_{m} r_{o}^{2} \omega^{2} s^{4} \sin \beta}{G_{3}(s)} F_{1}(s) \\
& +\frac{\eta_{13} r_{m} r_{o}^{2} \omega^{2} s^{4} \cos \beta}{G_{3}(s)} F_{2}(s)+\frac{\omega^{2}}{\left(s^{2}+\omega_{\psi}^{2}\right)} F_{3}(s) \\
& -\frac{\eta_{13} r_{m} r_{o}^{2}\left(\omega^{2} s^{4}+\omega_{x}^{2} \omega^{2} s^{2}\right)}{G_{3}(s)} F_{4}(s),
\end{aligned}
$$

$\Phi(s)$

$$
\begin{aligned}
= & \frac{s^{2} \omega^{2} \sin \beta F_{1}(s)}{G_{2}(s)}-\frac{s^{2} \omega^{2} \cos \beta F_{2}(s)}{G_{2}(s)} \\
& +\frac{\left(s^{2}+\omega_{x}^{2}\right) \omega^{2} F_{4}(s)}{G_{2}(s)},
\end{aligned}
$$

where $G_{1}(s)=\left(s^{2}+\omega_{1}^{2}\right)\left(s^{2}+\omega_{x}^{2}\right)^{2}-\left(\eta_{13}+\eta_{23}\right) r_{m}\left(s^{2}+\omega_{x}^{2}\right) s^{4}$, $G_{2}(s)=\left(s^{2}+\omega_{1}^{2}\right)\left(s^{2}+\omega_{x}^{2}\right)-\left(\eta_{13}+\eta_{23}\right) r_{m} s^{4}$, and $G_{3}(s)=(1+$ $\left.\eta_{13} r_{m} r_{o}^{2}\right)\left(s^{2}+\omega_{x}^{2}\right)^{2}\left(s^{2}+\omega_{1}^{2}\right)-\left(1+\eta_{13} r_{m} r_{o}^{2}\right)\left(s^{6}+\omega_{x}^{2} s^{4}\right)\left(\eta_{13}+\eta_{23}\right) r_{m}$.

Applying the inverse Laplace transformation to (22), introduce frequency ratio $n_{x}, n_{y}, n_{\psi}$, and $n_{1}$ into the formulas:

$$
\begin{aligned}
& n_{x}=\frac{\omega}{\omega_{x}}, \\
& n_{y}=\frac{\omega}{\omega_{y}}, \\
& n_{\psi}=\frac{\omega}{\omega_{\psi}}, \\
& n_{1}=\frac{\omega}{\omega_{1}} .
\end{aligned}
$$

In this case, the spring stiffness values $k_{x}, k_{y}, k_{\psi}$, and $k_{1}$ are converted into the function of frequency ratios $n_{x}, n_{y}, n_{\psi}$, and $n_{1}$ through the natural frequency, respectively. In the paper, we assume that $\omega_{x} \approx \omega_{y} \approx \omega_{\psi}$, and so it follows that $n_{x} \approx$ $n_{y} \approx n_{\psi}$. From (22) and (23) one gets

$$
\left[\begin{array}{l}
x \\
y \\
\psi \\
\varphi_{1}
\end{array}\right]=\left[\begin{array}{llll}
\mu_{11} & \mu_{12} & \mu_{13} & \mu_{14} \\
\mu_{21} & \mu_{22} & \mu_{23} & \mu_{24} \\
\mu_{31} & \mu_{32} & \mu_{33} & \mu_{34} \\
\mu_{41} & \mu_{42} & \mu_{43} & \mu_{44}
\end{array}\right]\left[\begin{array}{l}
f_{1}(t) \\
f_{2}(t) \\
f_{3}(t) \\
f_{4}(t)
\end{array}\right],
$$

where

$$
\begin{aligned}
& \mu_{11} \\
& =\frac{\left(\eta_{13}+\eta_{23}\right) r_{m} n_{1}^{2} n_{x}^{4} \sin ^{2} \beta}{-\left(n_{x}^{2}-1\right)^{2}\left(n_{1}^{2}-1\right)+n_{x}^{2} n_{1}^{2}\left(n_{x}^{2}-1\right)\left(\eta_{13}+\eta_{23}\right) r_{m}} \\
& \quad+\frac{n_{x}^{2}}{\left(1-n_{x}^{2}\right)}
\end{aligned}
$$

$\mu_{12}$

$$
\begin{aligned}
& =\frac{-\left(\eta_{13}+\eta_{23}\right) r_{m} n_{1}^{2} n_{x}^{4} \cos \beta \sin \beta}{-\left(n_{x}^{2}-1\right)^{2}\left(n_{1}^{2}-1\right)+n_{x}^{2} n_{1}^{2}\left(n_{x}^{2}-1\right)\left(\eta_{13}+\eta_{23}\right) r_{m}} \\
& \mu_{13}=0, \\
& \mu_{14} \\
& =\frac{\left(\eta_{13}+\eta_{23}\right) r_{m}\left(-n_{x}^{4} n_{1}^{2}+n_{1}^{2} n_{x}^{2}\right) \sin \beta}{-\left(n_{x}^{2}-1\right)^{2}\left(n_{1}^{2}-1\right)+n_{x}^{2} n_{1}^{2}\left(n_{x}^{2}-1\right)\left(\eta_{13}+\eta_{23}\right) r_{m}},
\end{aligned}
$$

$\mu_{21}$

$$
=\frac{-\left(\eta_{13}+\eta_{23}\right) r_{m} n_{1}^{2} n_{x}^{4} \sin \beta \cos \beta}{-\left(n_{x}^{2}-1\right)^{2}\left(n_{1}^{2}-1\right)+n_{x}^{2} n_{1}^{2}\left(n_{x}^{2}-1\right)\left(\eta_{13}+\eta_{23}\right) r_{m}},
$$

$\mu_{22}$

$$
\begin{aligned}
= & \frac{\left(\eta_{13}+\eta_{23}\right) r_{m} n_{1}^{2} n_{x}^{4} \cos ^{2} \beta}{-\left(n_{x}^{2}-1\right)^{2}\left(n_{1}^{2}-1\right)+n_{x}^{2} n_{1}^{2}\left(n_{x}^{2}-1\right)\left(\eta_{13}+\eta_{23}\right) r_{m}} \\
& +\frac{n_{y}^{2}}{1-n_{y}^{2}},
\end{aligned}
$$

$\mu_{23}=0$

$\mu_{24}$

$$
=\frac{\left(\eta_{13}+\eta_{23}\right) r_{m}\left(n_{1}^{2} n_{x}^{4}-n_{1}^{2} n_{x}^{2}\right) \cos \beta}{-\left(n_{x}^{2}-1\right)^{2}\left(n_{1}^{2}-1\right)+n_{x}^{2} n_{1}^{2}\left(n_{x}^{2}-1\right)\left(\eta_{13}+\eta_{23}\right) r_{m}},
$$

$\mu_{31}$

$$
=\frac{-\eta_{13} r_{m} r_{0}^{2} n_{1}^{2} n_{\psi}^{4} \sin \beta}{-\left(n_{x}^{2}-1\right)^{2}\left(n_{1}^{2}-1\right)+n_{x}^{2} n_{1}^{2}\left(n_{x}^{2}-1\right)\left(\eta_{13}+\eta_{23}\right) r_{m}},
$$

$\mu_{32}$

$$
=\frac{\eta_{13} r_{m} r_{0}^{2} n_{1}^{2} n_{\psi}^{4} \cos \beta}{-\left(n_{x}^{2}-1\right)^{2}\left(n_{1}^{2}-1\right)+n_{x}^{2} n_{1}^{2}\left(n_{x}^{2}-1\right)\left(\eta_{13}+\eta_{23}\right) r_{m}},
$$

$\mu_{33}=\frac{n_{\psi}^{2}}{l_{0}\left(-n_{\psi}^{2}+1\right)}$

$\mu_{34}$

$$
=\frac{\eta_{13} r_{m} r_{0}^{2}\left(n_{1}^{2} n_{\psi}^{4}-n_{1}^{2} n_{\psi}^{2}\right)}{-\left(n_{x}^{2}-1\right)^{2}\left(n_{1}^{2}-1\right)+n_{x}^{2} n_{1}^{2}\left(n_{x}^{2}-1\right)\left(\eta_{13}+\eta_{23}\right) r_{m}},
$$

$\mu_{41}=\frac{-n_{1}^{2} n_{x}^{2} \sin \beta}{\left(n_{x}^{2}-1\right)\left(n_{1}^{2}-1\right)-n_{x}^{2} n_{1}^{2}\left(\eta_{13}+\eta_{23}\right) r_{m}}$,

$\mu_{42}=\frac{n_{1}^{2} n_{x}^{2} \cos \beta}{\left(n_{x}^{2}-1\right)\left(n_{1}^{2}-1\right)-n_{x}^{2} n_{1}^{2}\left(\eta_{13}+\eta_{23}\right) r_{m}}$, 
TABLE 1: Parameter values.

(a) Parameter values for system equation (13)

\begin{tabular}{lccc}
\hline Unbalanced rotor & Vibrobody & Pendulum rod & Induction motor \\
\hline$r=0.05[\mathrm{~m}]$ & $l_{e}=0.3[\mathrm{~m}]$ & $l=0.3 \sim 0.9[\mathrm{~m}]$ & $m_{1}=15[\mathrm{~kg}]$ \\
$m_{2}=0.6 \sim 3[\mathrm{~kg}]$ & $m_{0}=100[\mathrm{~kg}]$ & $k_{1}=4980 \sim 24402510[\mathrm{~N} / \mathrm{rad}]$ & $L_{m i}=0.13[\mathrm{H}]$ \\
$m_{3}=3[\mathrm{~kg}]$ & $k_{x}=246490000 \sim 50307[\mathrm{~N} / \mathrm{m}]$ & $f_{1}=15[\mathrm{Nm} /(\mathrm{rad} / \mathrm{s})]$ & $L_{s i}=0.1[\mathrm{H}]$ \\
$\omega_{m}=152 \sim 157[\mathrm{rad} / \mathrm{s}]$ & $f_{x}=1064[\mathrm{Ns} / \mathrm{m}]$ & $\beta=0, \pi / 6, \pi / 4, \pi / 3[\mathrm{rad}]$ & $n_{p}=2$ \\
- & $k_{y}=246490000 \sim 50307[\mathrm{~N} / \mathrm{m}]$ & & $R_{r i}=0.54[\Omega]$ \\
- & $f_{y}=1064[\mathrm{Ns} / \mathrm{m}]$ & - & $U_{S 0}=220[\mathrm{~V}]$ \\
- & $k_{\psi}=50307 \sim 246490000[\mathrm{~N} / \mathrm{m}]$ & - & - \\
- & $f_{\psi}=1064[\mathrm{Nm} /(\mathrm{rad} / \mathrm{s})]$ & & - \\
\hline
\end{tabular}

(b) Parameter values according to dimensionless equation (19)

$$
\begin{gathered}
\eta_{13}=5 \\
\eta_{23}=0.3 \sim 1 \\
r_{m}=0.025 \\
n_{x} \approx n_{y} \approx n_{\psi}=0.2 \sim 7.0 \\
n_{1}=0.2 \sim 7 \\
r_{o}=1 \sim 3 \\
r_{l}=0.08,0.17
\end{gathered}
$$

$\mu_{43}=0$,

$$
\mu_{44}=\frac{\left(n_{1}^{2} n_{x}^{2}-n_{1}^{2}\right)}{\left(n_{x}^{2}-1\right)\left(n_{1}^{2}-1\right)-n_{x}^{2} n_{1}^{2}\left(\eta_{13}+\eta_{23}\right) r_{m}} .
$$

Parameter $\mu_{i j}(i=1,2,3,4, j=1,2,3,4)$ in (25) represents the mutual coupling coefficients among the rotors, the pendulum rod, and the vibrobody through the springs. The larger the value of the coupling coefficient is, the stronger the coupling ability of the system is. In order to understand the coupling characteristics of the system, the following numerical computations have been performed with assumption that parameters $\eta_{13}, \eta_{23}, l, r_{o}$, and $r_{m}$ are constants. In this case, we can confirm the value of the coupling coefficients when changing the value of parameters $n_{x}, n_{y}, n_{\psi}, n_{1}$, and $\beta$ within definite ranges, respectively. According to Table 1 , we can determine the value of the coupling coefficients.

From Figure 2 it follows that the value of the coupling coefficients depends on the values of parameters $n_{x}, n_{y}, n_{\psi}$, $n_{1}$, and $\beta$. In this figure, it can been seen that the peak value of the coupling coefficients is related to the frequency ratio of the system. However, frequency ratios $n_{x}, n_{y}, n_{\psi}$, and $n_{1}$ are the function of the spring stiffness values $k_{x}$, $k_{y}, k_{\psi}$, and $k_{1}$, respectively. So the values of the coupling coefficients are determined by the spring stiffness. Clearly, for $0.1<n_{x}, n_{y}, n_{\psi}<0.9$ and $n_{1}=0.2$ or 0.8 , the vibration frequency of the system is less than the eigen-frequency of the system, which is denoted as before-resonance system; in this case, the absolute values of $\mu_{i j}$ are smaller except $\mu_{33}$ and $\mu_{44}$ when $n_{1}=0.8$. For $0.9 \leq n_{x}, n_{y}, n_{\psi}<1.5$ and $n_{1}=1.5$, the vibration frequency of the system is approximately equal to the eigen-frequency of the system, which is denoted by resonance system; in this case, the absolute value of parameter $\mu_{i j}$ is far larger when $\beta$ is nonzero. For $n_{x}, n_{y}, n_{\psi} \geq 1.5$ and $n_{1}=3.0$, the vibration frequency of the system is larger than the eigen-frequency of the system, which is denoted by afterresonance system; in this case, the value of parameter $\mu_{i j}$ is no more than 2.0. Then, we can define type of the coupling system generally used in engineering according to frequency ratio, as shown in Table 2.

\section{Synchronization and Stability}

In this section, we analyze the synchronization and stability of the system with theoretical method. As was already mentioned in (3), the family of synchronous solutions is assumed as

$$
\begin{aligned}
& \varphi_{2}=\omega t+\alpha_{1}, \\
& \varphi_{3}=\omega t+\alpha_{2} .
\end{aligned}
$$

So in synchronous state, (24) can be rewritten as

$$
\begin{aligned}
x(t) & =\mu_{11}\left[\eta_{23} r_{m} r \cos \left(\omega t+\alpha_{1}\right)+r_{m} r \cos \left(\omega t+\alpha_{2}\right)\right] \\
+ & \mu_{12}\left[\eta_{23} r_{m} r \sin \left(\omega t+\alpha_{1}\right)+r_{m} r \sin \left(\omega t+\alpha_{2}\right)\right] \\
+ & \mu_{14} \frac{\eta_{23} r_{l}}{\left(\eta_{13}+\eta_{23}\right)} \sin \left(\omega t+\alpha_{1}-\beta\right), \\
y(t) & =\mu_{21}\left[\eta_{23} r_{m} r \cos \left(\omega t+\alpha_{1}\right)+r_{m} r \cos \left(\omega t+\alpha_{2}\right)\right] \\
& +\mu_{22}\left[\eta_{23} r_{m} r \sin \left(\omega t+\alpha_{1}\right)+r_{m} r \sin \left(\omega t+\alpha_{2}\right)\right] \\
& +\mu_{24} \frac{\eta_{23} r_{l}}{\left(\eta_{13}+\eta_{23}\right)} \sin \left(\omega t+\alpha_{1}-\beta\right),
\end{aligned}
$$



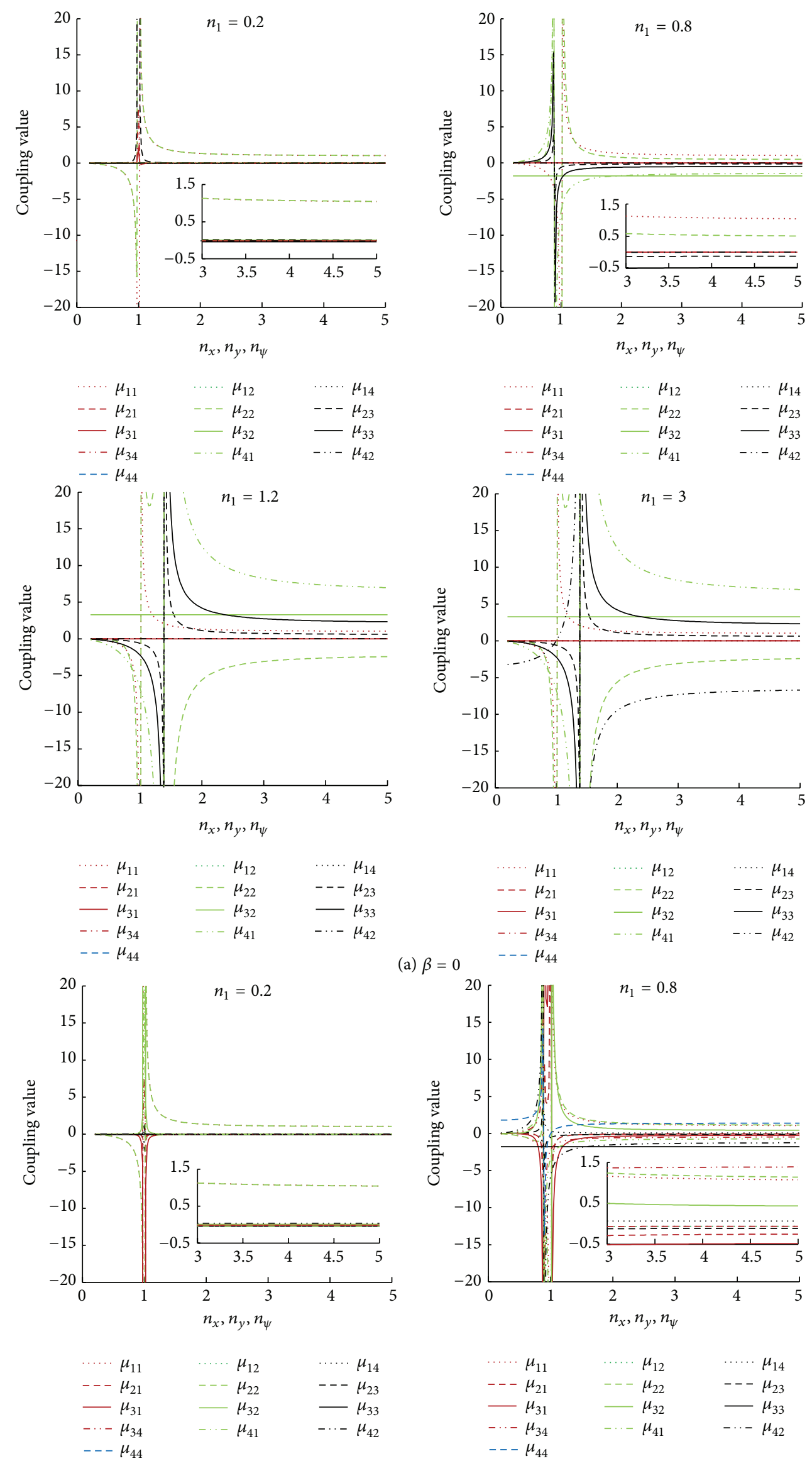

(a) $\beta=0$

$$
\begin{array}{lll}
\ldots . \cdot \mu_{11} & \cdots \cdots \mu_{12} & \cdots \cdots \mu_{14} \\
---\mu_{21} & ---\mu_{22} & ---\mu_{23} \\
-\mu_{31} & -\mu_{32} & -\mu_{33} \\
-\cdots-\mu_{34} & -\cdots-\mu_{41} & -\cdots-\mu_{42}
\end{array}
$$
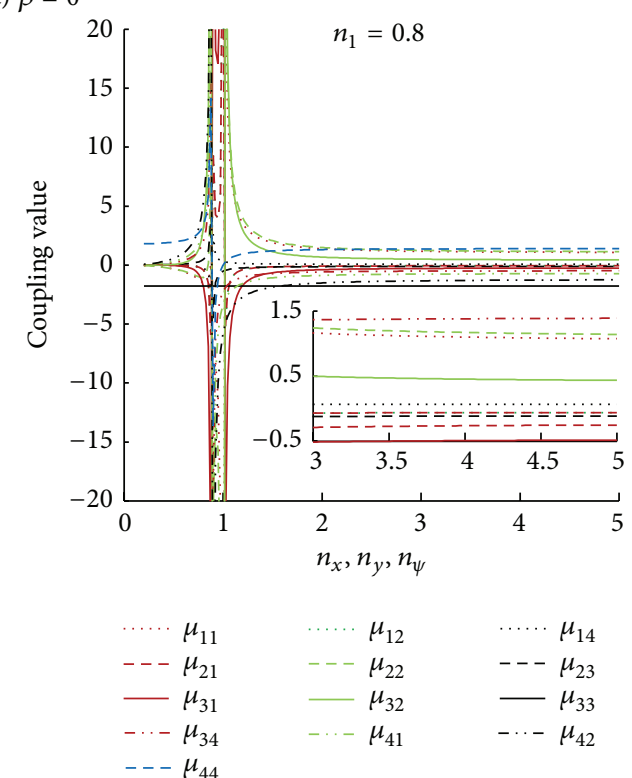

Figure 2: Continued. 

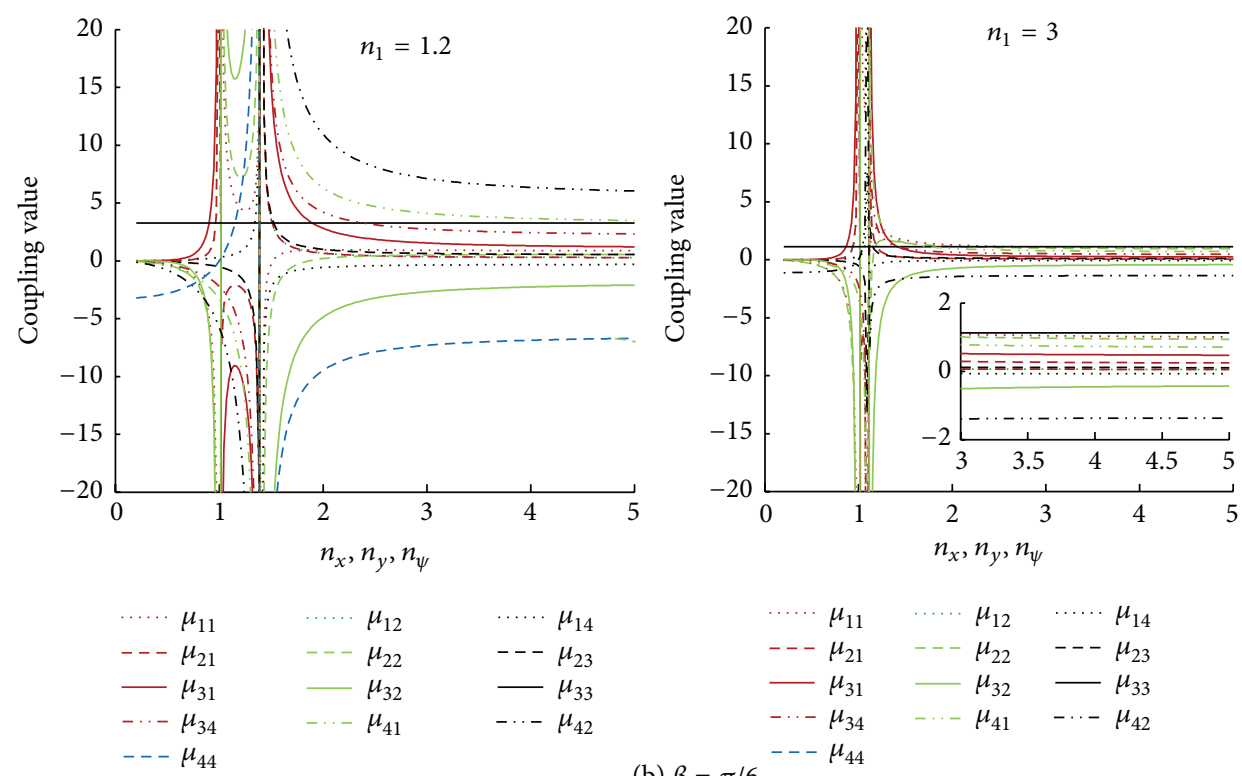

(b) $\beta=\pi / 6$
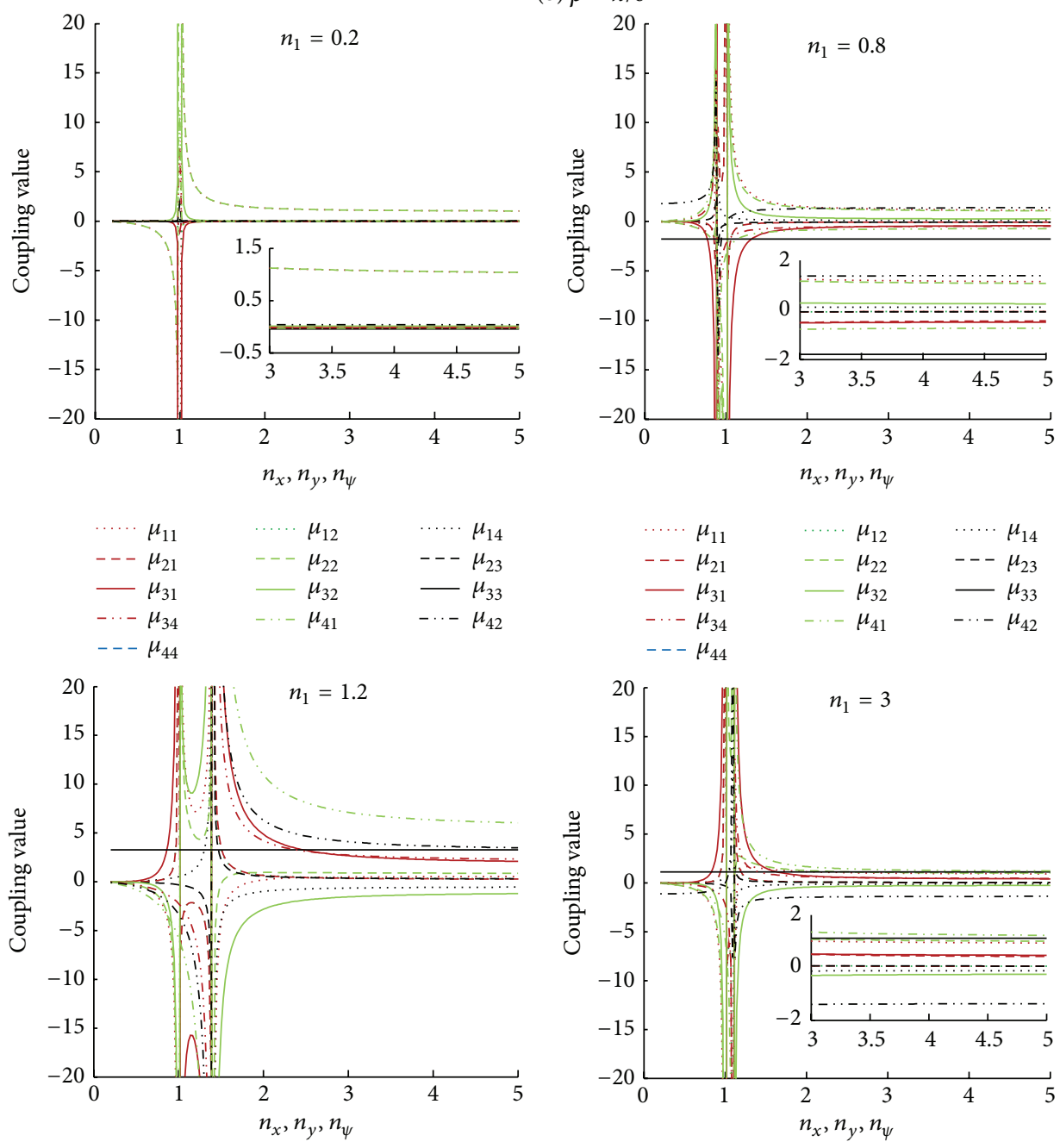

$$
\begin{array}{lll}
\cdots-\mu_{11} & \cdots \mu_{12} & \cdots \cdots \mu_{14} \\
---\mu_{21} & ---\mu_{22} & ---\mu_{23} \\
-\mu_{31} & -\mu_{32} & -\mu_{33} \\
-\cdots-\mu_{34} & -\cdots \mu_{41} & -\cdots-\mu_{42} \\
---\mu_{44} & &
\end{array}
$$

$\begin{array}{rlr}\cdots \cdots \mu_{11} & \cdots \cdot \mu_{12} & \cdots \cdots \mu_{14} \\ ---\mu_{21} & --\mu_{22} & ---\mu_{23} \\ -\mu_{31} & -\mu_{32} & -\mu_{33} \\ -\cdots-\mu_{34} & -\cdots-\mu_{41} & -\cdots-\mu_{42} \\ \text { (c) } \beta=\pi / 3^{---} \mu_{44} & & \end{array}$

FIGURE 2: The value of the coupling coefficients of the system for $\eta_{13}=5, \eta_{23}=1, r_{m}=0.025, l=0.6$, and $r_{o}=2$ considering the difference of angular $\beta$. 
TABLE 2: Type of the coupling system.

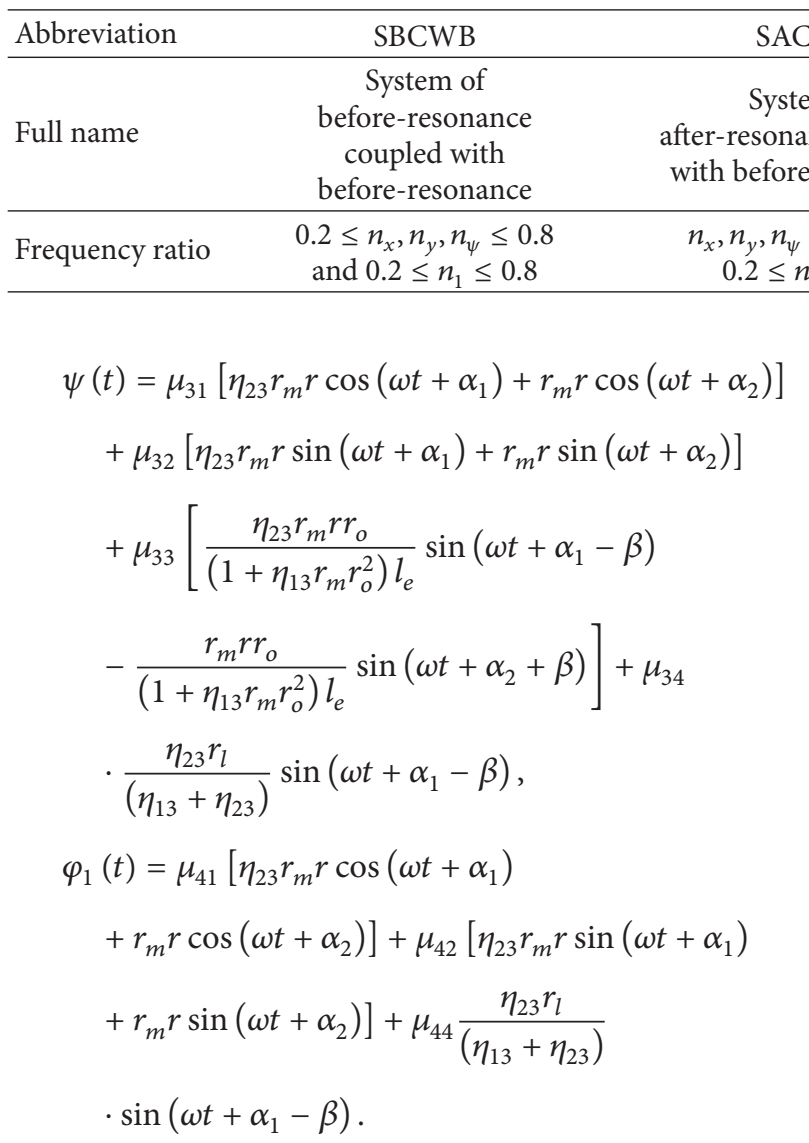

Specifying parameter $\alpha$ as the phase difference between the two rotors, we have

$$
\alpha=\alpha_{1}-\alpha_{2}
$$

Consequently, the basic equation (4) is expressed as

$$
\begin{aligned}
P_{1} & =\left\langle\mu \Phi_{1}\right\rangle=M_{e 1}-R_{e 1}-\frac{1}{2} \\
& \cdot m_{3} r^{2} \omega^{2}\left[\eta_{23} r_{m}\left(\mu_{11}+\mu_{22}\right) \sin \alpha\right. \\
& +\eta_{23} l r_{m}\left(\mu_{32}+\mu_{42}\right) \sin (\alpha-\beta) \\
& -\eta_{23} l r_{m}\left(\mu_{41}+\mu_{31}\right) \cos (\alpha-\beta) \\
& \left.-\frac{\eta_{23} \mu_{33} r_{o}^{2} r_{m}}{1+\eta_{13} r_{m} r_{o}^{2}} \sin (\alpha-2 \beta)\right]=0, \\
P_{2} & =\left\langle\mu \Phi_{2}\right\rangle=M_{e 2}-R_{e 2}-\frac{1}{2} \\
& \cdot m_{3} r^{2} \omega^{2}\left[-\eta_{23} r_{m}\left(\mu_{11}+\mu_{22}\right) \sin \alpha\right. \\
& -\left(\frac{\eta_{23} \mu_{24}}{\left(\eta_{13}+\eta_{23}\right) l}-\eta_{23} l r_{m} \mu_{32}\right) \sin (\alpha-\beta)
\end{aligned}
$$

$$
\begin{aligned}
& +\left(\frac{\eta_{23} \mu_{14}}{\left(\eta_{13}+\eta_{23}\right) l}+\eta_{23} l r_{m} \mu_{31}\right) \cos (\alpha-\beta) \\
& +\left(\frac{\eta_{23} r_{m} r_{o}^{2} \mu_{33}}{1+\eta_{13} r_{m} r_{o}^{2}}+\frac{\eta_{23} \mu_{34}}{\eta_{13}+\eta_{23}}\right) \sin (\alpha-2 \beta) \\
& \left.+\mu_{31} l r_{m} \cos \beta-\mu_{32} l r_{m} \sin \beta\right]=0 .
\end{aligned}
$$

During the synchronous state, consider the excessive torque $Z_{s}(\omega)$ of the rotors to be zero [1]:

$$
Z_{i}(\omega)=M_{e i}-R_{e i}=0, \quad(i=1,2)
$$

Therefore, according to (29) and (30), further calculations lead to the following form:

$$
\begin{aligned}
P_{1} & -P_{2}=-\left(\mu_{11}+\mu_{22}\right) \eta_{23} r_{m} \sin \alpha \\
& -\frac{1}{2}\left(\eta_{23} l r_{m} \mu_{42}+\frac{\eta_{23} \mu_{24}}{\left(\eta_{13}+\eta_{23}\right) l}\right) \sin (\alpha-\beta) \\
& +\left[\frac{1}{2} \mu_{41} \eta_{23} l r_{m}+\frac{1}{2} \frac{\eta_{23} \mu_{14}}{\left(\eta_{13}+\eta_{23}\right) l}+\mu_{31} \eta_{23} l r_{m}\right] \\
\cdot & \cos (\alpha-\beta)+\left(\frac{\eta_{23} \mu_{33} r_{o}^{2} r_{m}}{1+\eta_{13} r_{m} r_{o}^{2}}+\frac{1}{2} \frac{\eta_{23} \mu_{34}}{\eta_{13}+\eta_{23}}\right) \\
\cdot & \sin (\alpha-2 \beta)+\frac{1}{2} \mu_{31} l r_{m} \cos \beta-\frac{1}{2} \mu_{32} l r_{m} \sin \beta \\
= & 0 .
\end{aligned}
$$

We can define (31) as the balance equation of synchronization state of the system, on which we can determine phase difference $\alpha$ with numerical computations. It should be noted that the value of the phase difference is related to parameters $\eta_{13}, \eta_{23}, l, \beta, r_{o}, r_{l}, r_{m}$, and $\mu_{i j}$. However, parameter $\mu_{i j}$ is the function mainly related to frequency ratios $n_{x}, n_{y}, n_{\psi}$, $n_{1}, l$, and $\beta$, which indicate that the spring stiffness and the structure parameters of the system are the key parameters to determine the value of phase difference $\alpha$.

Now, let us consider the stability of the synchronous rotation for the rotors. Considering (31), the following criterion of synchronous stability is obtained from (6): 


$$
\begin{aligned}
\chi= & \frac{\partial\left(P_{1}-P_{2}\right)}{\partial \alpha_{1}}=\frac{\partial\left(P_{1}-P_{2}\right)}{\partial \alpha} \\
& =-m_{3} r^{2} \omega^{2}\left[\eta_{23} r_{m}\left(\mu_{11}+\mu_{22}\right) \cos \alpha\right. \\
& +\frac{1}{2}\left(\eta_{23} l r_{m} \mu_{42}+\frac{\eta_{23} \mu_{24}}{\left(\eta_{13}+\eta_{23}\right) l}\right) \cos (\alpha-\beta) \\
& +\left(\frac{1}{2} \eta_{23} l r_{m} \mu_{41}+\frac{1}{2} \frac{\eta_{23} \mu_{14}}{\left(\eta_{13}+\eta_{23}\right) l}+\mu_{31} \eta_{23} l r_{m}\right) \\
& \cdot \sin (\alpha-\beta)-\left(\frac{\eta_{23} \mu_{33} r_{o}^{2} r_{m}}{1+\eta_{13} r_{m} r_{o}^{2}}+\frac{1}{2} \frac{\eta_{23} \mu_{34}}{\eta_{13}+\eta_{23}}\right) \\
& \cdot \cos (\alpha-2 \beta)]<0 .
\end{aligned}
$$

Rearranging (32), the stability criterion of synchronization of the system can be simplified as

$$
\begin{aligned}
& \left(\mu_{11}+\mu_{22}\right) \eta_{23} r_{m} \cos \alpha \\
& +\frac{1}{2}\left(\eta_{23} l r_{m} \mu_{42}+\frac{\eta_{23} \mu_{24}}{\left(\eta_{13}+\eta_{23}\right) l}\right) \cos (\alpha-\beta) \\
& +\left(\frac{1}{2} \mu_{41} \eta_{23} l r_{m}+\frac{1}{2} \frac{\eta_{23} \mu_{14}}{\left(\eta_{13}+\eta_{23}\right) l}+\mu_{31} \eta_{23} l r_{m}\right) \\
& \cdot \sin (\alpha-\beta)-\left(\frac{\eta_{23} \mu_{33} r_{o}^{2} r_{m}}{1+\eta_{13} r_{m} r_{o}^{2}}+\frac{1}{2} \frac{\eta_{23} \mu_{34}}{\eta_{13}+\eta_{23}}\right) \\
& \cdot \cos (\alpha-2 \beta)>0 .
\end{aligned}
$$

Only should the values of the system parameters satisfy the balance equation and the stability criterion of synchronization of the system, the synchronous state of the rotors can be determined. In this case, the phase difference between the rotors is called stable phase difference.

\section{Numerical Discussions}

The abovementioned sections have given some theoretical discussions in the simplified form on synchronization problem for the vibration system that the two unbalanced rotors coupled with a pendulum rod. In this section, we will quantitatively analyze the results of the stable phase difference. The parameter values corresponding to general engineering application are as given in Table 1.

5.1. Theoretical Solutions. Under the condition that the balance equation and stability criterion of synchronization between the two rotors (see (31) and (33)) are satisfied, the stable phase difference can be determined by using numerical method. It is worthy to point out that the motion type of the rigid vibrobody is dependent on the synchronous state, which is determined by the values of the stable phase difference. Based on the theoretical deductions, numerical computation will be conducted in the following sections, considering

the variations of installation angular $\beta$, frequency ratios $n_{x}$, $n_{y}, n_{\psi}$, and $n_{1}$, mass ratio $\eta_{23}$, and pendulum length $l$.

5.1.1. Synchronous State for SBCWB and SACWB. Depending on variations of the values of the system parameters, in this subsection, we observe the synchronous states of two different systems, that is, system of before-resonance coupled with before-resonance (SBCWB), and system of after-resonance coupled with before-resonance (SACWB). We choose the systems as the comparison of the synchronous states because we want to know whether the variations of the stiffness coefficients of the springs have influence on the synchronous states, although SBCWB may be rarely applied in vibration screening engineering.

In Figure 3, we present the values of the stable phase difference for SBCWB and SACWB considering variation of frequency ratio and installation angular $\beta$. The values of the structure parameters are fixed at $\eta_{13}=5, \eta_{23}=1, r_{o}=2$, $r_{l}=0.08$, and $l=0.6$; additionally, frequency ratio $n_{1}$ is set as $0.2,0.4,0.6$, and 0.8 , respectively. From the figure, the stable phase difference locates in the different values in the intervals of $0.2 \leq n_{x}, n_{y}, n_{\psi} \leq 0.8$ and $1.5 \leq n_{x}, n_{y}, n_{\psi} \leq 7$, respectively. It demonstrates that the stiffness coefficients of the springs determine the synchronous states in some certain extent ([1] describes that the motion, as the existence of $\alpha \in(-\pi / 2, \pi / 2)$, is called synphase synchronization and the motion, as the existence of $\alpha \in(\pi / 2,3 \pi / 2)$, is called antiphase synchronization). For SBCWB, the values of the phase difference stabilize at the region of synphase synchronization. On the contrary, for SACWB, the values of the phase difference stabilize at the region of antiphase synchronization. Further observations were as follows: the smaller the value of frequency ratio $n_{1}$ is, with adjusting installation angular $\beta$, the weaker the variation of the phase difference is. However, frequency ratios are inversely proportional to the stiffness coefficient of the springs; in this case, we can believe that extremely large value of stiffness coefficient of the torque spring leads to disadvantage of adjustment of the synchronous state.

For synchronization of asymmetric mass of the two rotors (i.e., considering $\eta_{23} \neq 1.0$ ), (31) is the balanced equation when the system is operated in the synchronous state, and we can find that mass ratio $\eta_{23}$ of the unbalanced rotors in this equation cannot be removed. Whether it effects the synchronous state of SBCWB and SACWB depends on our following numerical computation. From Figures 4(a)$4(\mathrm{~d})$, it follows the stable phase difference considering the asymmetric mass of the two rotors. In this case, we suppose that the values of the other structure parameters are fixed at $\eta_{13}=5, \eta_{23}=0.4, r_{o}=2, r_{l}=0.08$, and $l=0.6$, in addition, frequency ratio $n_{1}$ is ascertained at $n_{1}=0.2$, $n_{1}=0.4, n_{1}=0.6$, and $n_{1}=0.8$, respectively. According to the date label in the figure, it can be seen that the values of the stable phase are less varying when changing the mass of the rotor in the pendulum rod. Therefore, the asymmetric mass of the two rotors has little influence on the synchronous state in the pendulum-rotor system.

On the other hand, whether length $l$ of the pendulum rod affects the synchronous state of the system will be discussed here. From Figure 5 it depicts the phase difference 

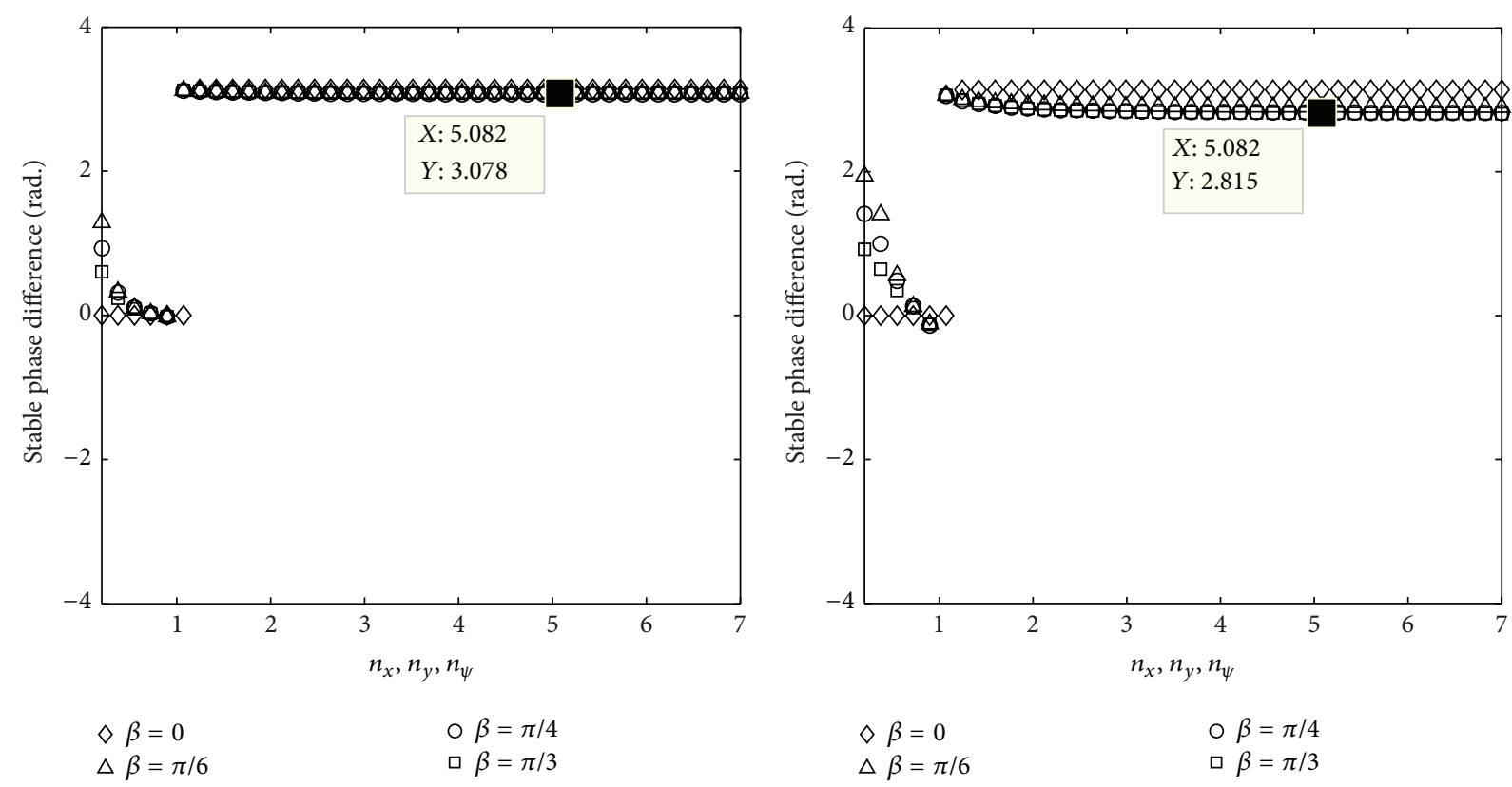

(a) $n_{1}=0.2$

(b) $n_{1}=0.4$

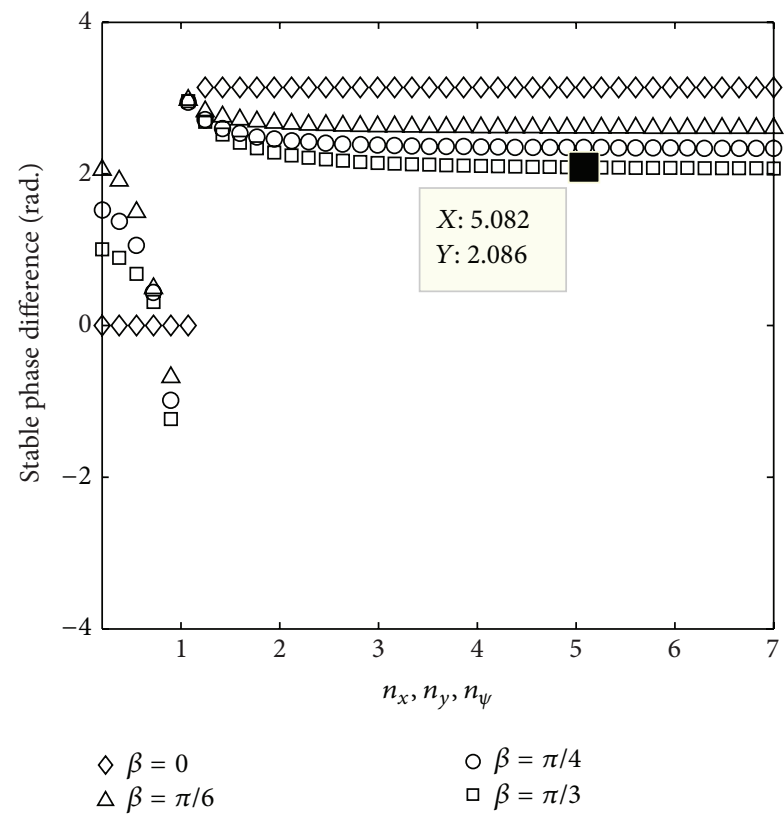

(c) $n_{1}=0.6$

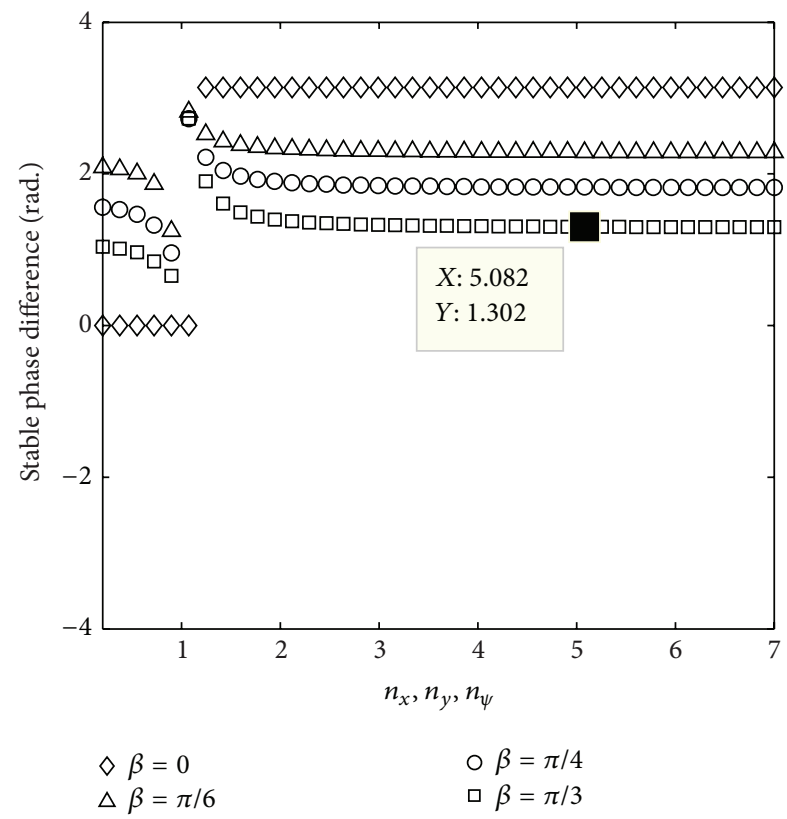

(d) $n_{1}=0.8$

FIGURE 3: Stable phase difference for SBCWB and SACWB considering variation of frequency ratio $n_{1}$ with $\eta_{13}=5, \eta_{23}=1, r_{o}=2, r_{l}=0.08$, and $l=0.6$ : (a) $n_{1}=0.2$, (b) $n_{1}=0.4$, (c) $n_{1}=0.6$, and (d) $n_{1}=0.8$.

for SACWB considering the length of the pendulum rod. Here, the values of the structure parameters of system are identical with Figure 3 except pendulum length $l$; additionally, frequency ratios $n_{x}, n_{y}, n_{\psi}$ are set as $n_{x}, n_{y}, n_{\psi}=3$, $n_{x}, n_{y}, n_{\psi}=4, n_{x}, n_{y}, n_{\psi}=5$, and $n_{x}, n_{y}, n_{\psi}=6$, respectively. According to the date label in the figure, it can be seen that the values of the stable phase are little varying when changing the value of installation angular $\beta$ of the pendulum rod, on the condition that the frequency ratio $n_{1}$ is small. In other words, if the value of stiffness coefficient of the torque spring, between the pendulum rod and the vibrobody, is very large in SACWB, the variation of installation angular $\beta$ weakly changes the synchronous state of the system.

5.1.2. Synchronous State for SBCWA and SACWA. In the above subsection, we have computed the stable phase difference for SBCWB and SACWB considering variations of the structure parameters. Here, we will concern the phase difference for the system of before-resonance coupled with after-resonance (SBCWA, i.e., $0.2 \leq n_{x}, n_{y}, n_{\psi} \leq 0.8$, and 

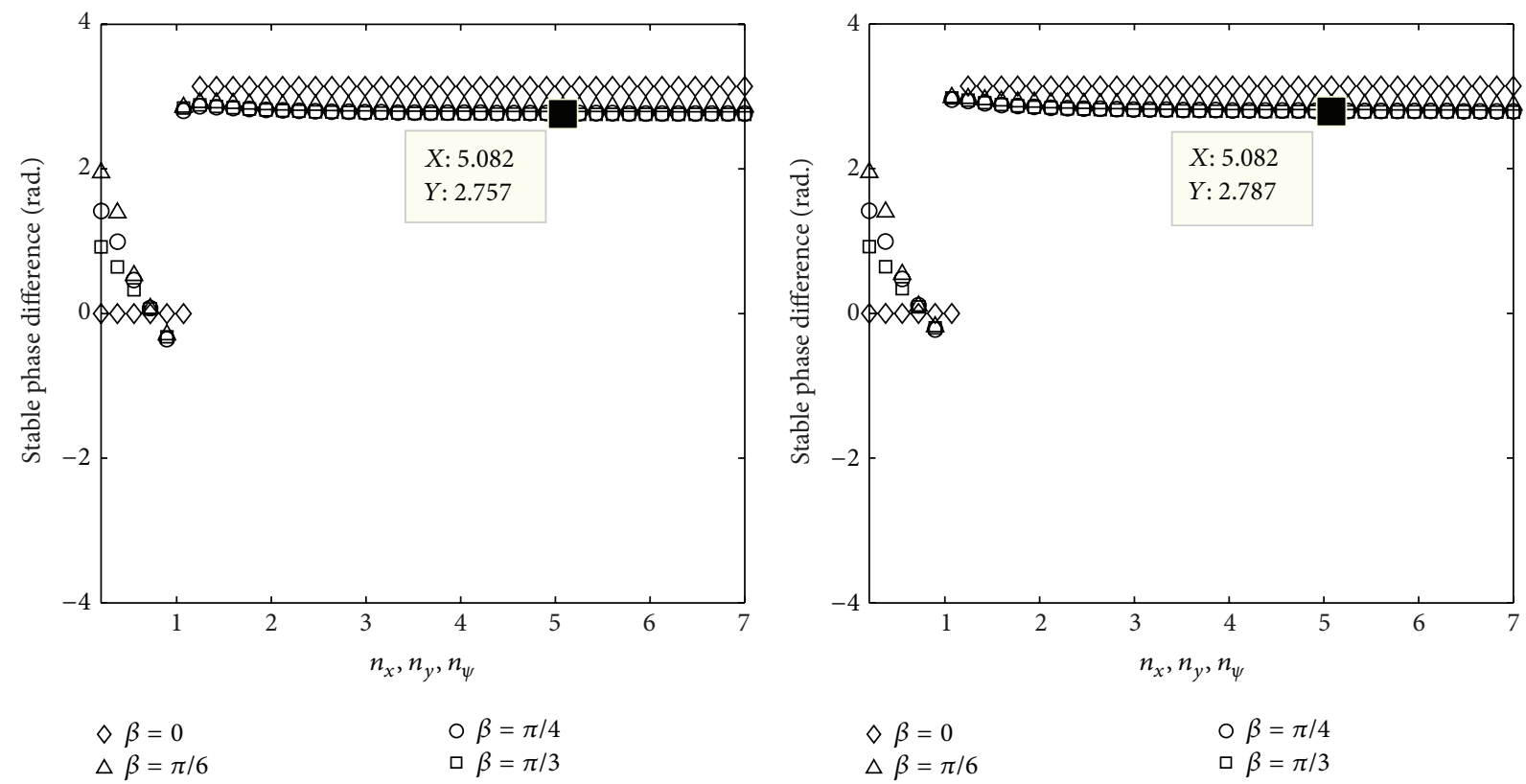

(a) $\eta_{23}=0.2$

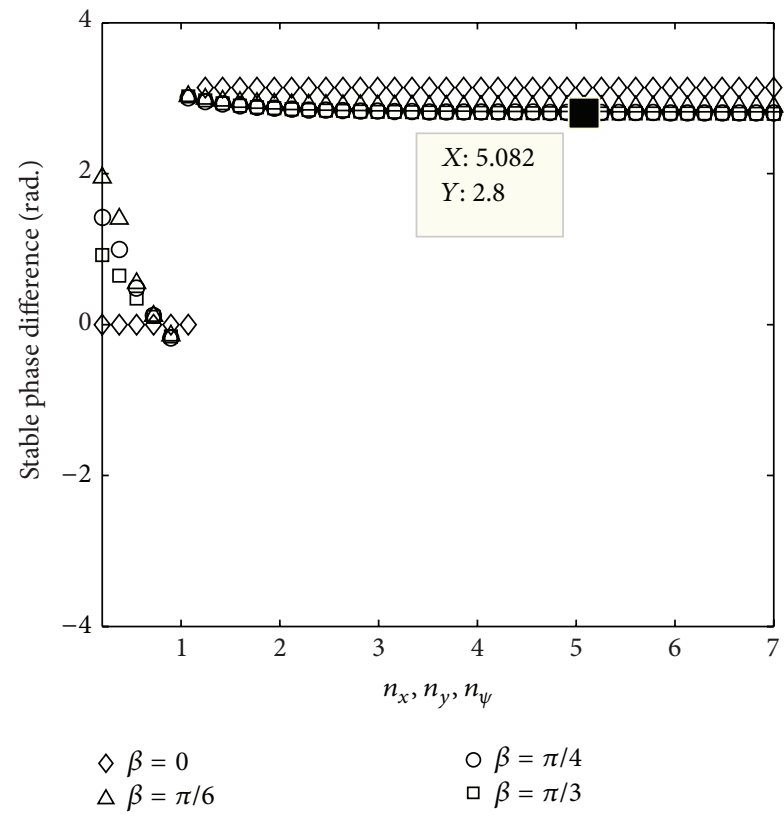

(c) $\eta_{23}=0.6$

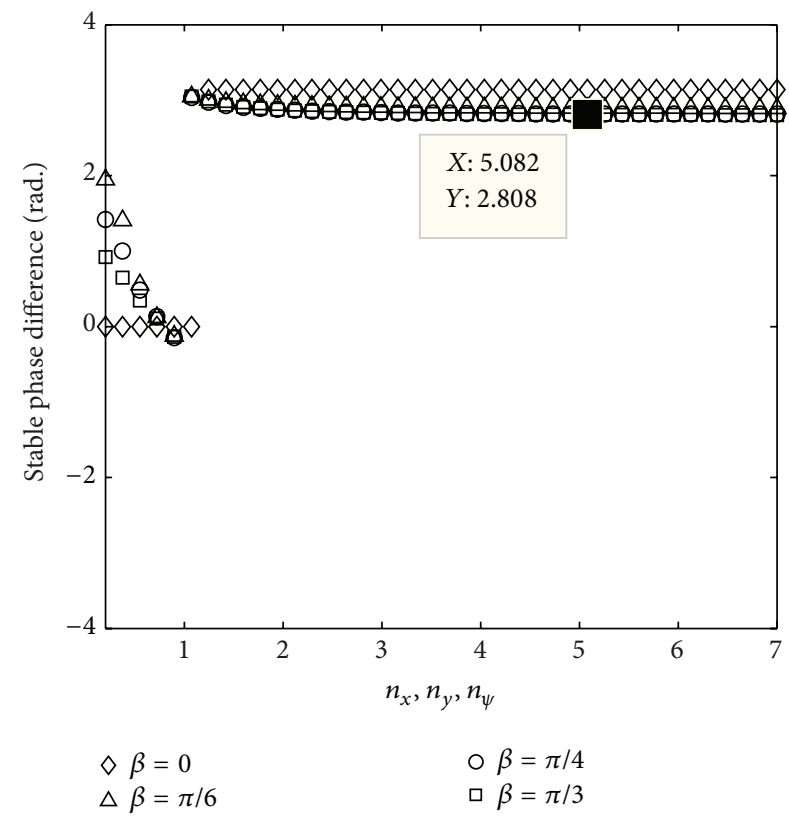

(d) $\eta_{23}=0.8$

FIGURE 4: Stable phase difference for SBCWB and SACWB considering asymmetric mass of the rotors with $\eta_{13}=5, n_{1}=0.4, r_{o}=2, r_{l}=0.08$, and $l=0.6$ : (a) $\eta_{23}=0.2$, (b) $\eta_{23}=0.4$, (c) $\eta_{23}=0.6$, and (d) $\eta_{23}=0.8$.

$n_{1} \geq 2.0$ ), and system of after-resonance coupled with afterresonance (SACWA, i.e., $n_{x}, n_{y}, n_{\psi} \geq 2.0$, and $n_{1} \geq 2.0$ ).

In Figure 6, we show the values of the stable phase difference for SBCWA and SACWA, assuming the variations of installation angular $\beta$ and frequency ratios $n_{x}, n_{y}, n_{\psi}$. The values of the other structure parameters are fixed at $\eta_{13}=5$, $\eta_{23}=1, r_{o}=2, r_{l}=0.08$, and $l=0.6$; moreover, frequency ratio $n_{1}$ is set as $n_{1}=3, n_{1}=4, n_{1}=5$, and $n_{1}=6$, respectively. According to the value of the stable phase difference, we can ascertain the synchronous characteristics of the systems.
It can be seen that, with increasing the frequency ratio $n_{1}$, the value of the phase difference is almost absent to change. Here, antiphase and synphase synchronization appearing in SBCWA and SACWA are merely dependent on the values of frequency ratio $n_{x}, n_{y}, n_{\psi}$ and installation angular $\beta$.

From Figure 7, it follows the phase difference for SACWA considering the asymmetric mass of the rotors; that is, $\eta_{23}=$ $0.2, \eta_{23}=0.4, \eta_{23}=0.6$, and $\eta_{23}=0.8$. Meanwhile, the values of the other physics parameters are fixed at $\eta_{13}=5$, $n_{1}=5, r_{o}=2, r_{l}=0.08$, and $l=0.6$. According to the date 


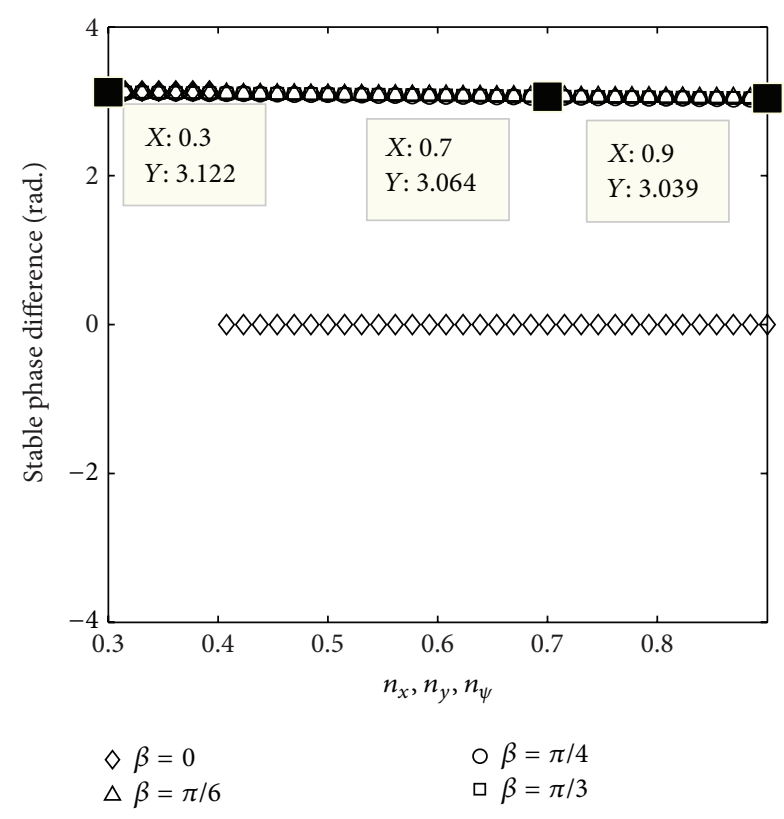

(a) $n_{1}=0.2$

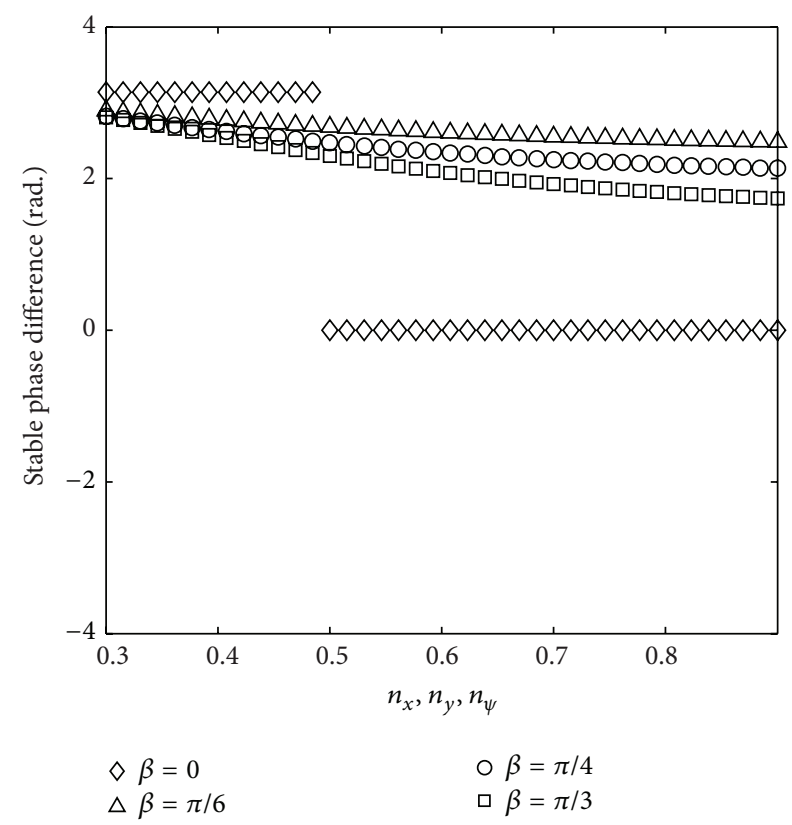

(c) $n_{1}=0.6$

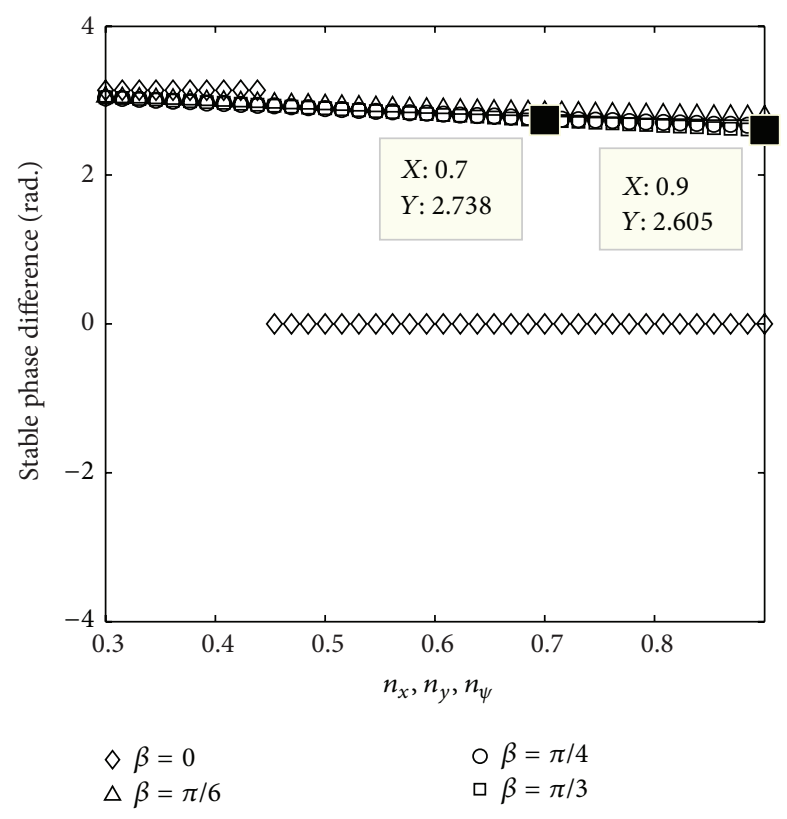

(b) $n_{1}=0.4$

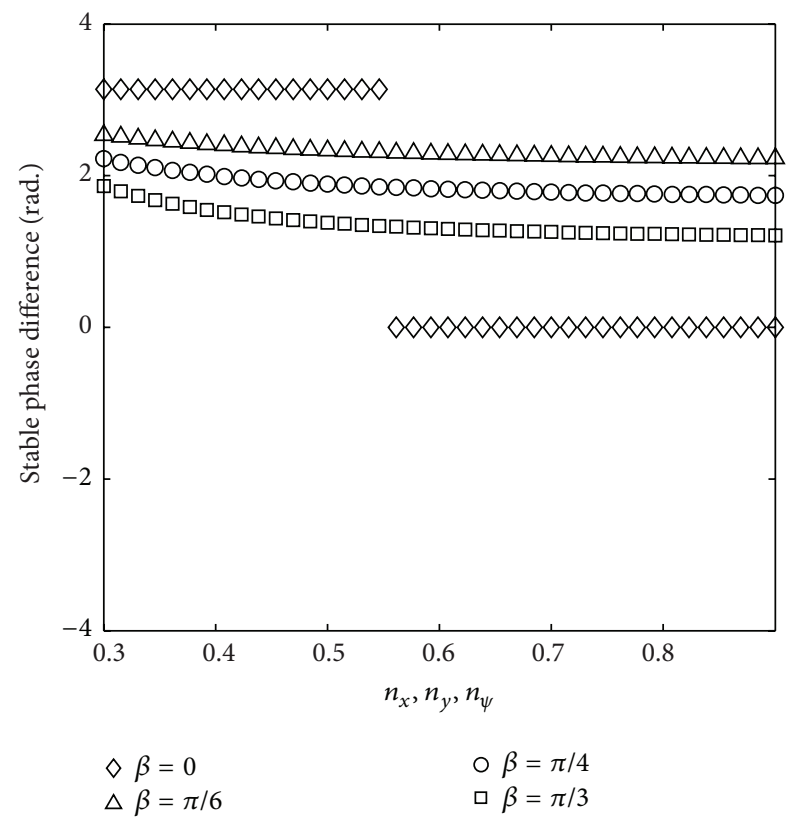

(d) $n_{1}=0.8$

FIGURE 5: Stable phase difference for SACWB considering the variation of length $l$ of pendulum rod with $\eta_{13}=5, \eta_{23}=1, n_{x} \approx n_{y} \approx n_{\psi}=5.0$, $r_{l}=0.08$, and $l_{e}=0.3$ : (a) $n_{1}=0.2$, (b) $n_{1}=0.4$, (c) $n_{1}=0.6$, and (d) $n_{1}=0.8$.

label in the figure, it can be seen that, the same as the above subsection, the values of the stable phase are varying a little when changing the mass of the rotor in the pendulum rod.

Finally, the variations of the phase difference for SACWA are compared in Figure 8, based on the variation of pendulum length $l$ and frequency ratio $n_{1}$ of the torque spring. In the numerical computations, the values of the other structure parameters are set the same as Figure 5 except $n_{1}=3, n_{1}=$ $4, n_{1}=5$, and $n_{1}=6$. For the synchronous state of the SACA, the phase difference from antiphase synchronization is gradually closed to the synphase synchronization with increasing the length of the pendulum. But for the variation of frequency ratio $n_{1}$, it can be seen that, for SACWA, the values of the stable phase are varying a little when changing the value of frequency ratio $n_{1}$.

5.2. Sample Verifications. Further analyses have been performed by computer simulations to verify our theoretical solutions above, which can be carried out by applying the Runge-Kutta routine with adaptive stepsize control to the 


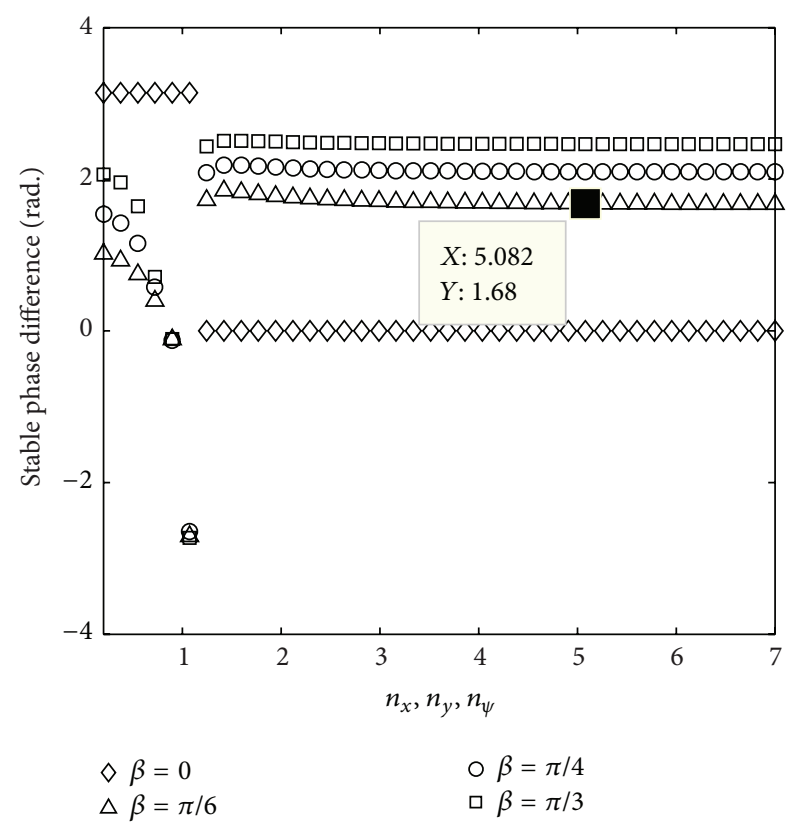

(a) $n_{1}=3$

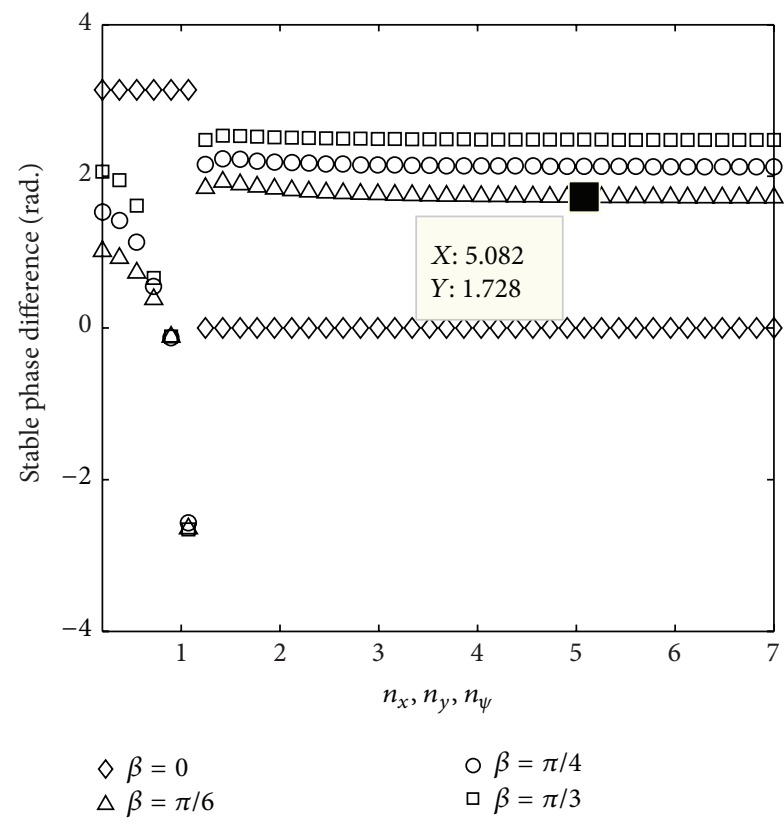

(c) $n_{1}=5$

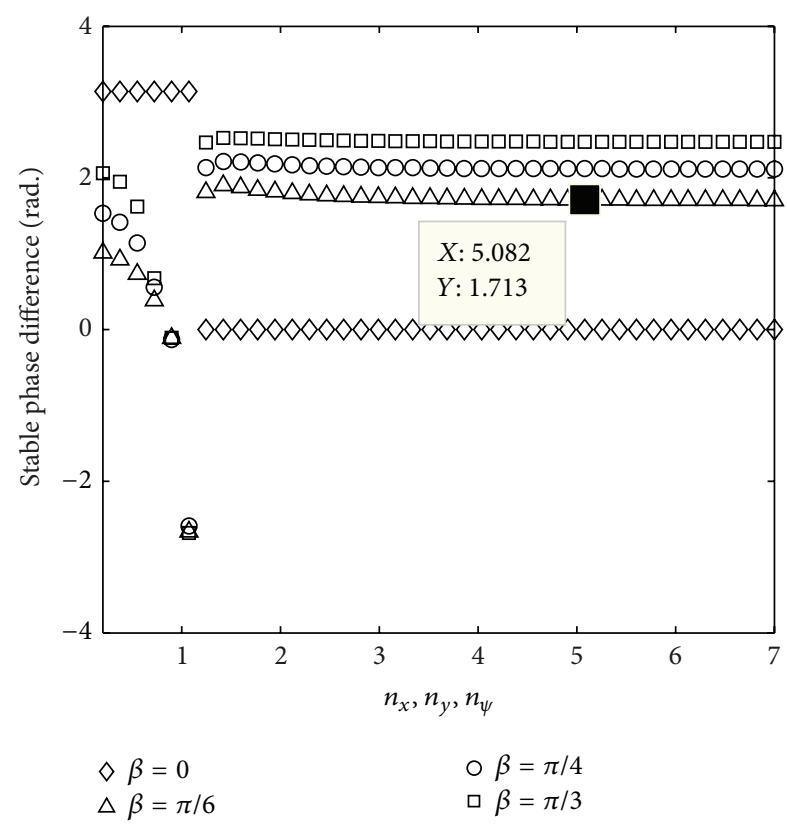

(b) $n_{1}=4$

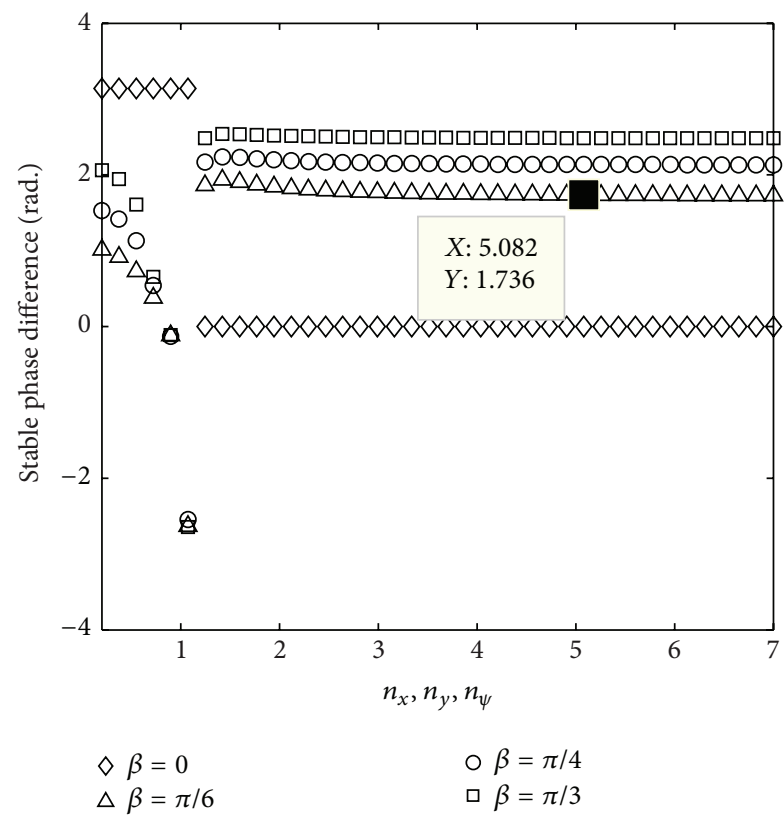

(d) $n_{1}=6$

Figure 6: Stable phase difference for SBCWA and SACWA considering $\eta_{13}=5, \eta_{23}=1, r_{o}=2, r_{l}=0.08$, and $l=0.6$ : (a) $n_{1}=3$, (b) $n_{1}=4$, (c) $n_{1}=5$, and (d) $n_{1}=6$.

dynamics equation (13). Here, the parameters of the two motors, which supplied the power source at the same time, are assumed to be identical.

5.2.1. Synchronization for $S A C W B$. Simulation results for SACWB, assuming that $\eta_{13}=5, \eta_{13}=1, n_{1}=0.4, n_{x} \approx$ $n_{y} \approx n_{\psi}=5.0, r_{o}=2.0, l=0.6$, and $\beta=\pi / 6$, are shown in Figure 9. When the two motors supplied the electric source at the same time, the angular accelerations of the two rotors are approximately identical as the inertia moments of the two rotors are identical and the spring stiffness is stronger (see Figure 9(a)). During the staring process of the system, the velocity difference existing between the two rotors leads the phase difference to be unstable. However, when the angular velocities of the motors reach the operation value and the pendulum rod oscillates steadily, the synchronization phenomenon occurs. At this moment, the coupling torques of the two rotors are approximated to $0.42[\mathrm{~N} \cdot \mathrm{m}]$. Meanwhile, the two motors steadily rotate in antiphase synchronization with phase difference $3.0[\mathrm{rad}]$ (see Figure 9(b)). In this 

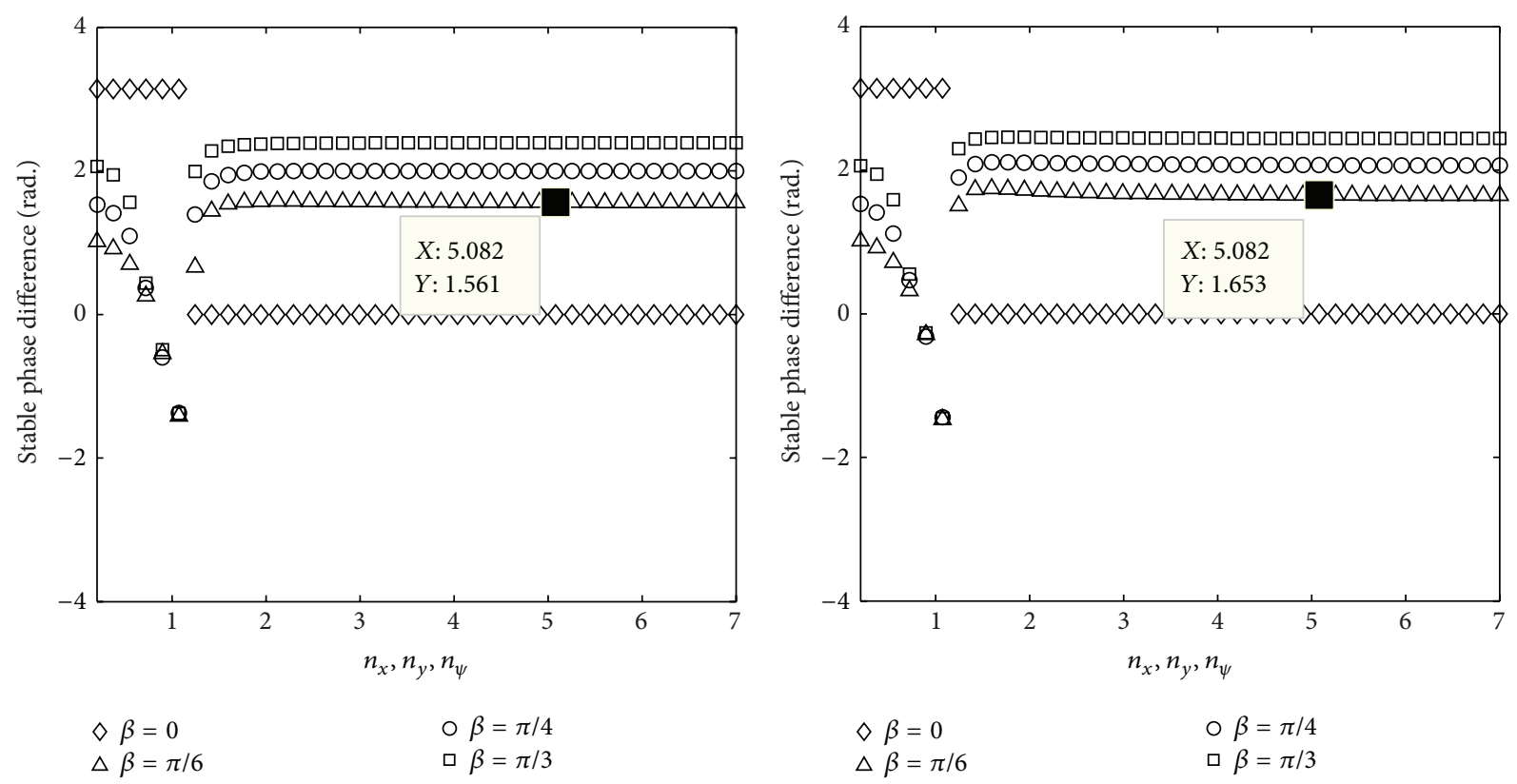

(a) $\eta_{23}=0.2$

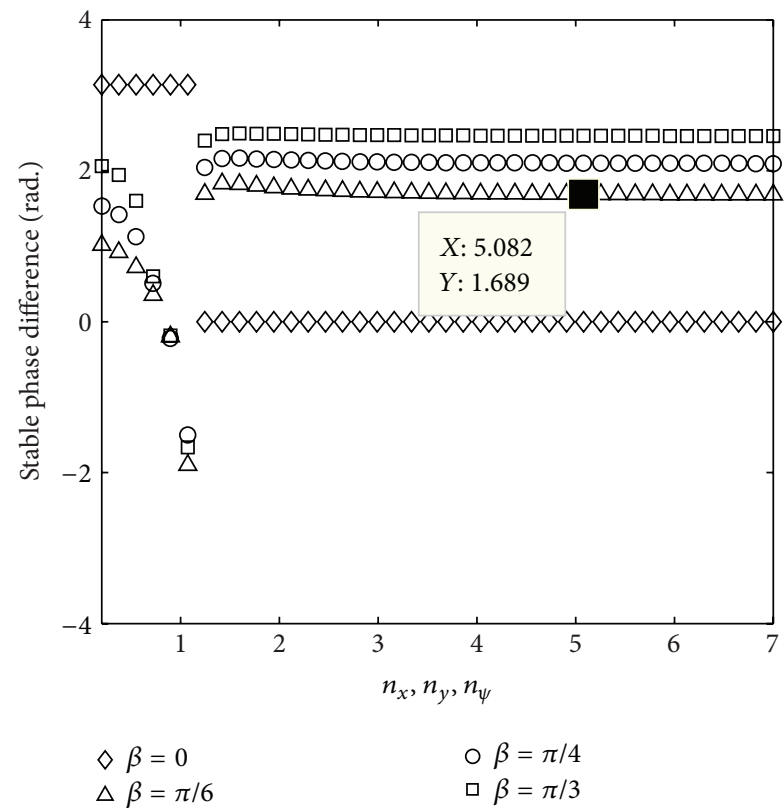

(c) $\eta_{23}=0.6$
○ $\beta=\pi / 4$

ㅁ $\beta=\pi / 3$ (b) $\eta_{23}=0.4$

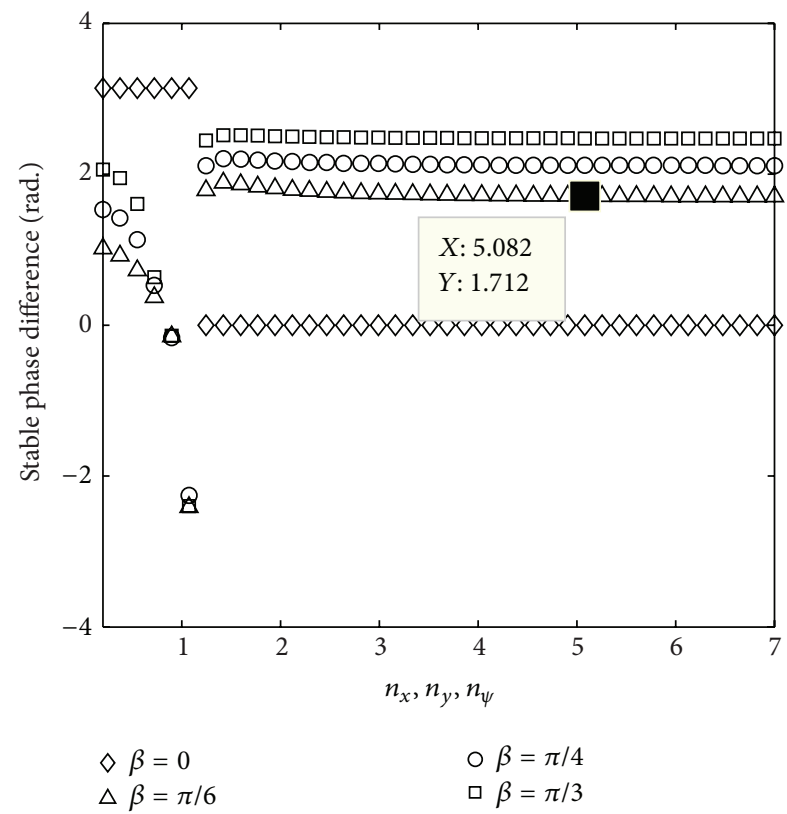

(d) $\eta_{23}=0.8$

FIGURE 7: Stable phase difference for SBCWA and SACWA assuming the nonidentical mass of rotors and considering $\eta_{13}=5, \eta_{23}=1, \eta_{1}=5$, $r_{o}=2, r_{l}=0.08$, and $l=0.6$ : (a) $\eta_{23}=0.2$, (b) $\eta_{23}=0.4$, (c) $\eta_{23}=0.6$, and (d) $\eta_{23}=0.8$.

synchronous state, the stable trajectory of mass center of the vibrobody in the case is a small ellipse because the centrifugal forces produced by the two rotors are counteracted. Therefore, the amplitudes of the stable responses of the vibrobody are small (see Figure 9(e)). From the displacement responses of the vibrobody in Figures 9(e) and 9(f), we can see that when the rotation velocity of the two rotors passes through the resonant region of the coupling system, the resonant responses of the vibrobody in the $x$-, $y$-, and $\psi$-direction appeared in the starting process except $\varphi_{1}$ - direction. The reason is that the natural frequency in $\varphi_{1}$-direction is larger than excitation frequency of the system. Comparing the value of the phase difference with that obtained by theoretical solution in Figure 3(b), it should be emphasized here that the simulation results are in agreement with the result obtained by the theoretical solutions (i.e., the stable phase difference 


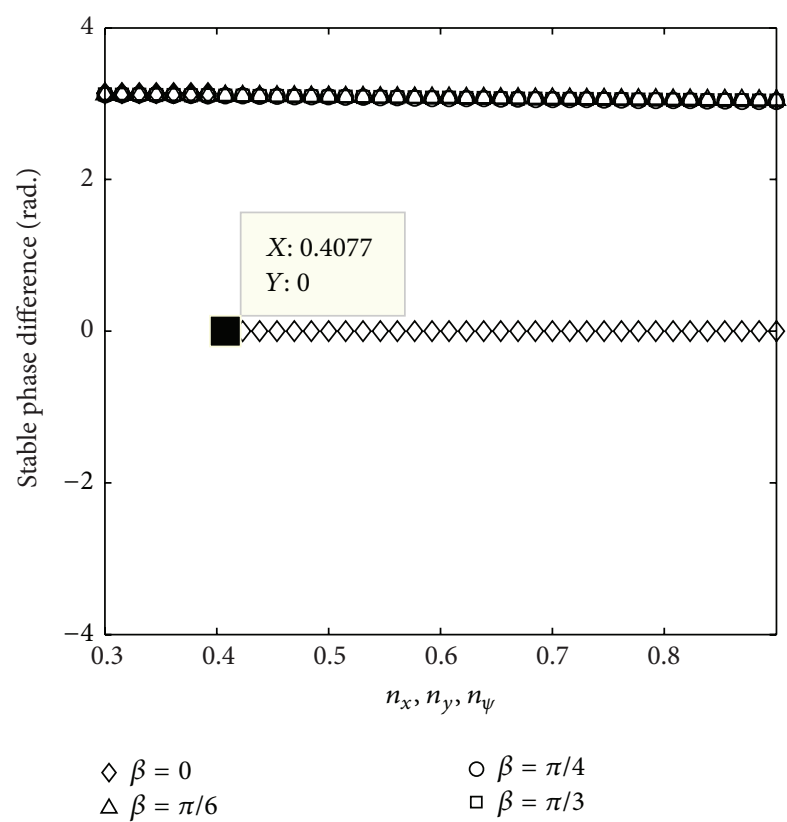

(a) $n_{1}=3$

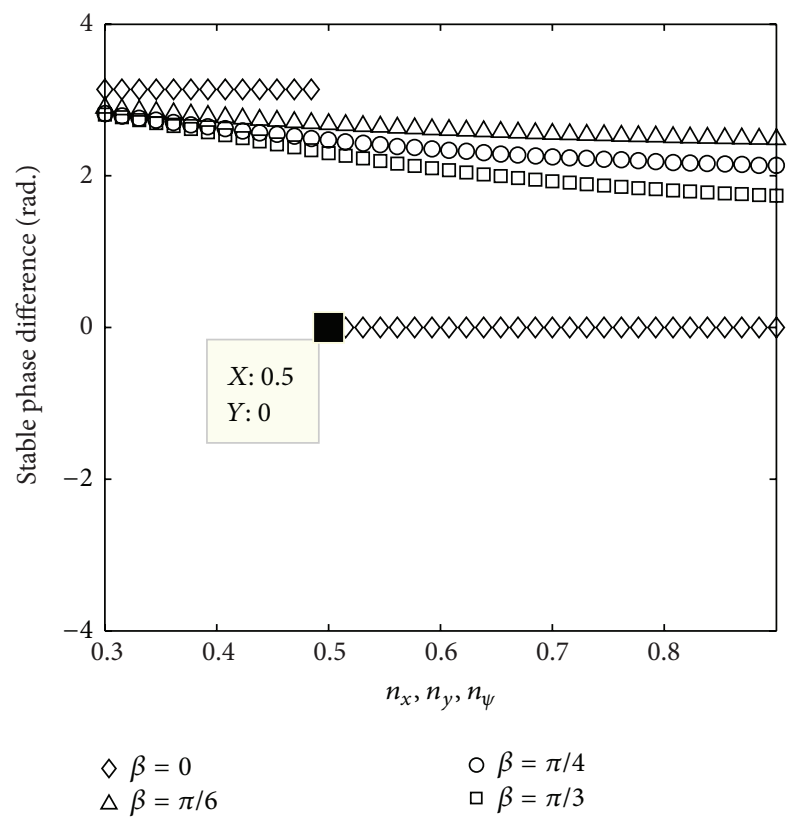

(c) $n_{1}=5$

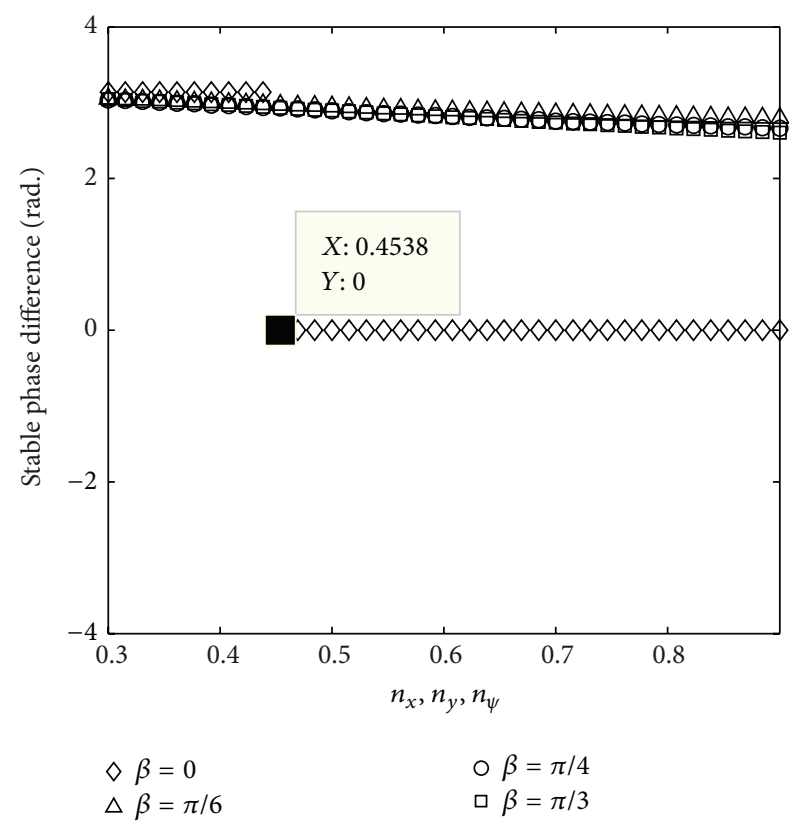

(b) $n_{1}=4$

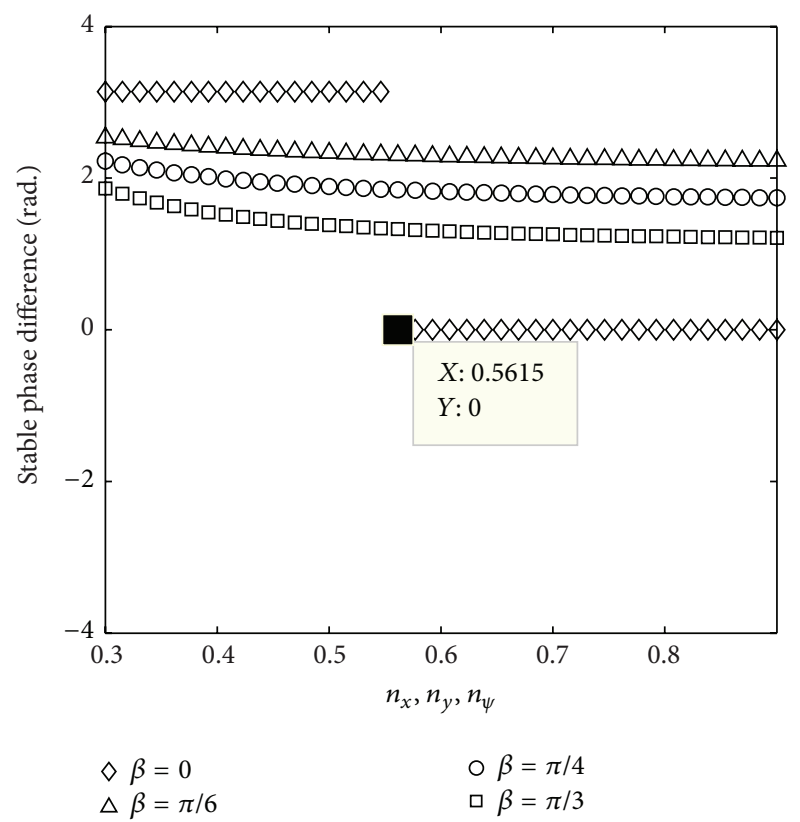

(d) $n_{1}=6$

FIGURE 8: Stable phase difference for SACWA assuming the nonidentical mass of rotors and considering $\eta_{13}=5, \eta_{23}=1, n_{x} \approx n_{y} \approx n_{\psi}=5.0$, $r_{l}=0.08$, and $l_{e}=0.3:$ (a) $n_{1}=3$, (b) $n_{1}=4$, (c) $n_{1}=5$, and (d) $n_{1}=6$.

with the theoretical calculation in Figure 3(b) is equal to $2.9[\mathrm{rad}]$; here the stable phase difference with the computer simulation is equal to 3.0 [rad]).

5.2.2. Synchronization for SACWA. Simulation results for SACWA, assuming that the values of system parameters are in accordance with Section 5.2.1 except $n_{1}=5$, are shown in Figure 10. When the two motors are supplied the electric source at the same time, the angular accelerations of the two rotors are nonidentical (see Figure 10(a)), and the reason can be attributed to the larger oscillation amplitude of pendulum (see Figure 10(e)). Then the angular velocities of the motors reach $154[\mathrm{rad} / \mathrm{s}]$, and the synchronization phenomenon occurs. At this moment, the coupling torques are approximated to $2.8[\mathrm{~N} \cdot \mathrm{m}]$ (see Figure 9(c)), and the phase difference stabilized at $1.78[\mathrm{rad}]$ (as $33.18[\mathrm{rad}]=(10 \pi+$ 1.78) [rad]). In the case, the synchronous state for SACWA can be considered the antiphase synchronization. In addition, the two motors steadily rotate in antiphase synchronization with phase difference 3.0 [rad] (see Figure 9(b)). In this case, 

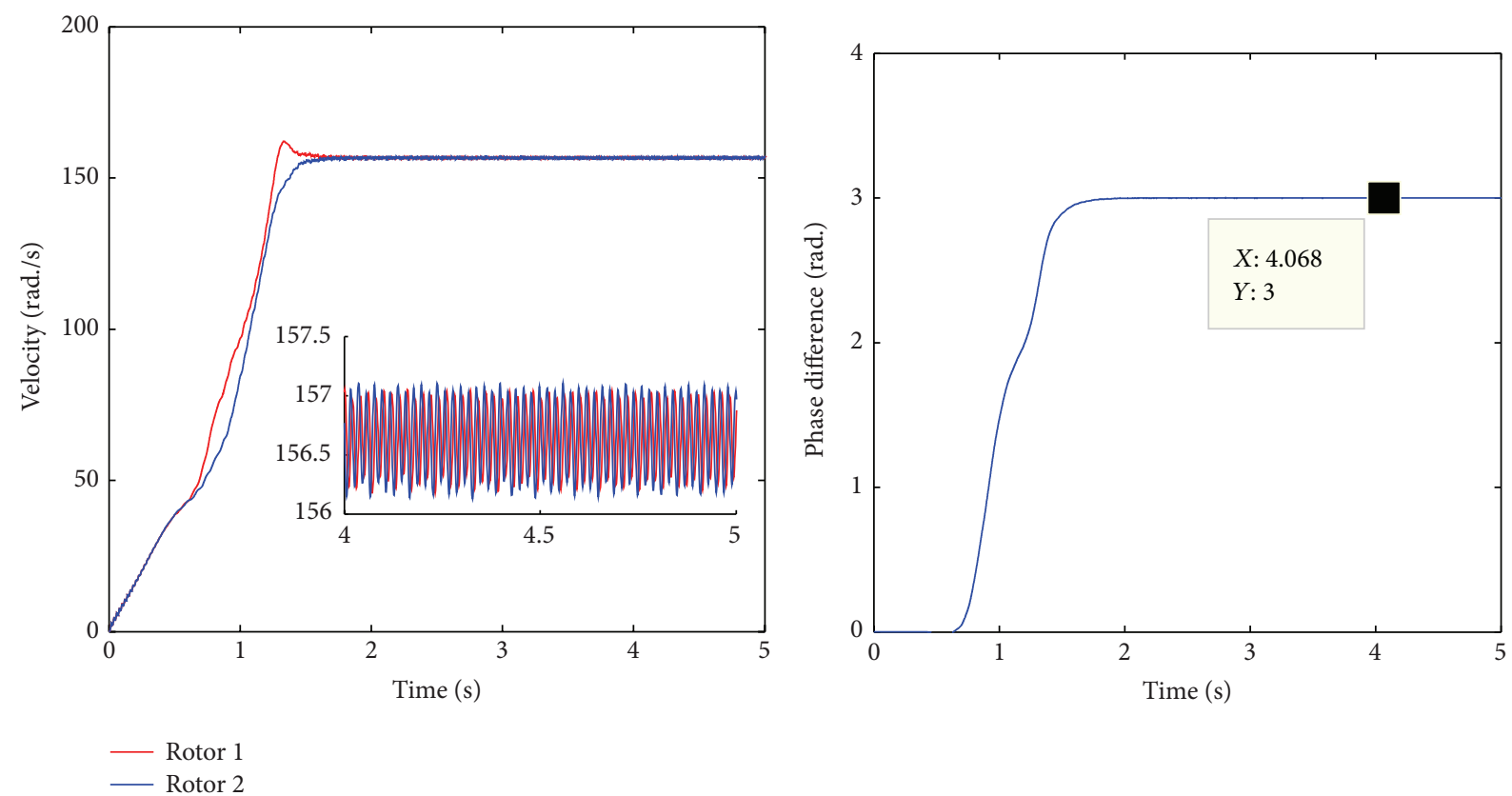

(a)

(b)
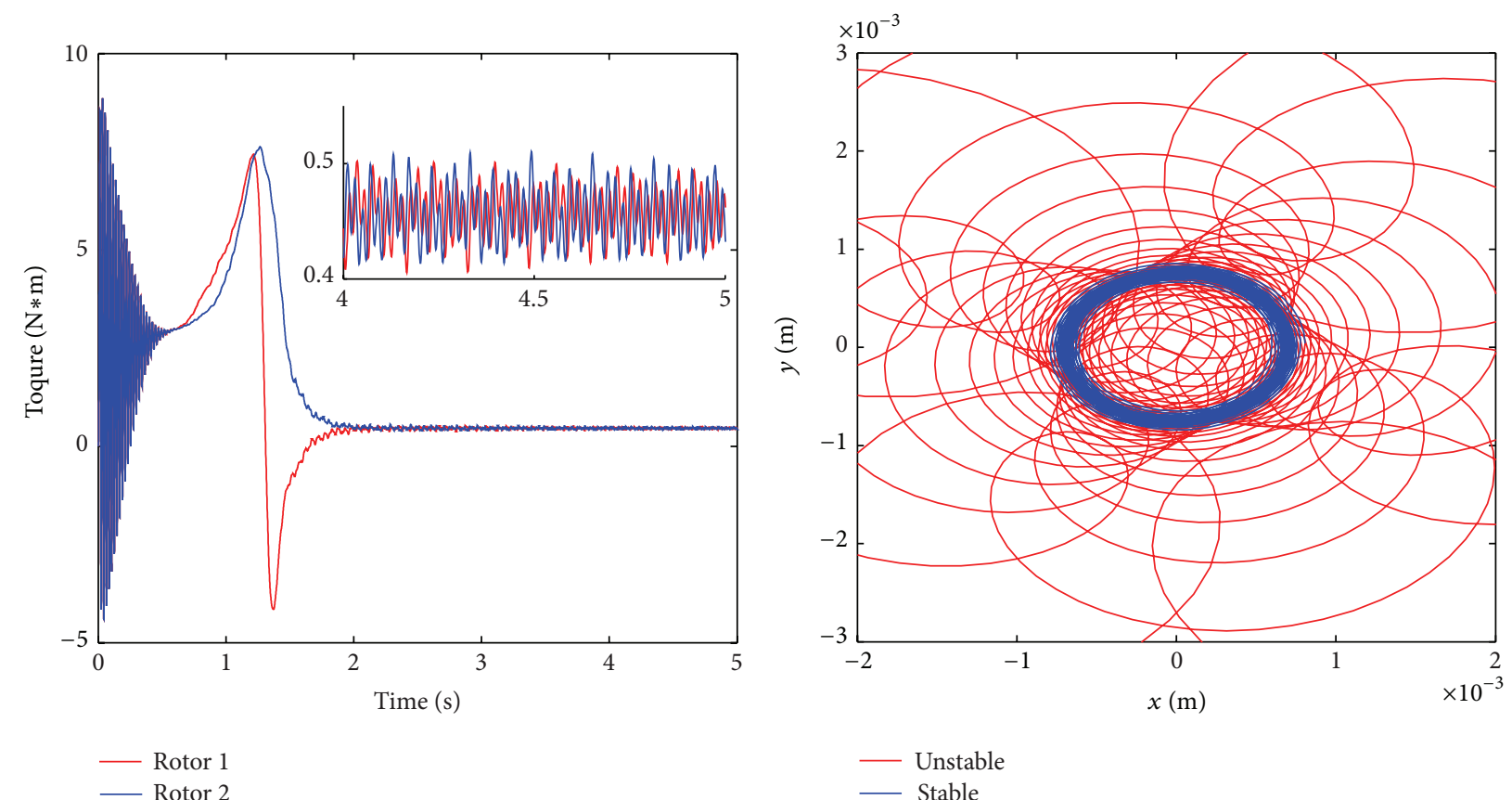

(c)
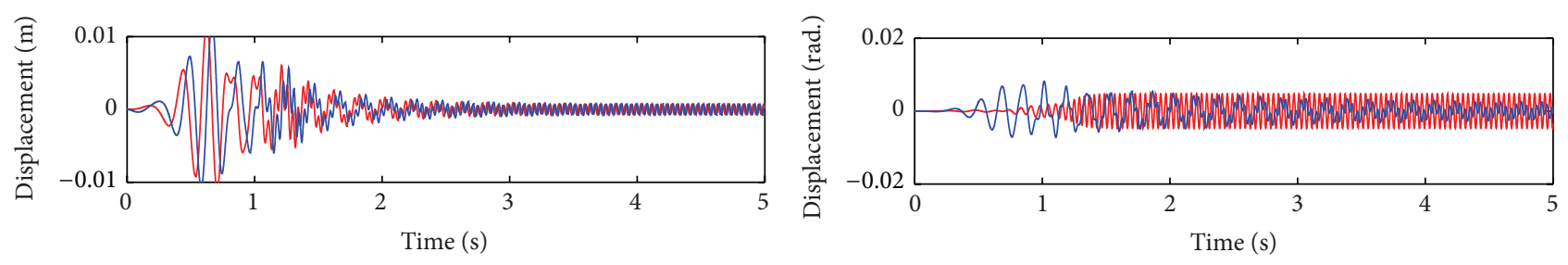

$-x$

$-\varphi_{1}$

(e)

(f)

FIGURE 9: Simulation results for SACWB: (a) rotational velocity, (b) phase difference of the two rotors, (c) torques of the two rotors, (d) trajectory of mass center of the vibrobody, (e) displacement responses of the vibrobody in $x$-and $y$-directions, and (f) displacement responses of the vibrobody in $\varphi_{1}$ - and $\psi$-directions. 


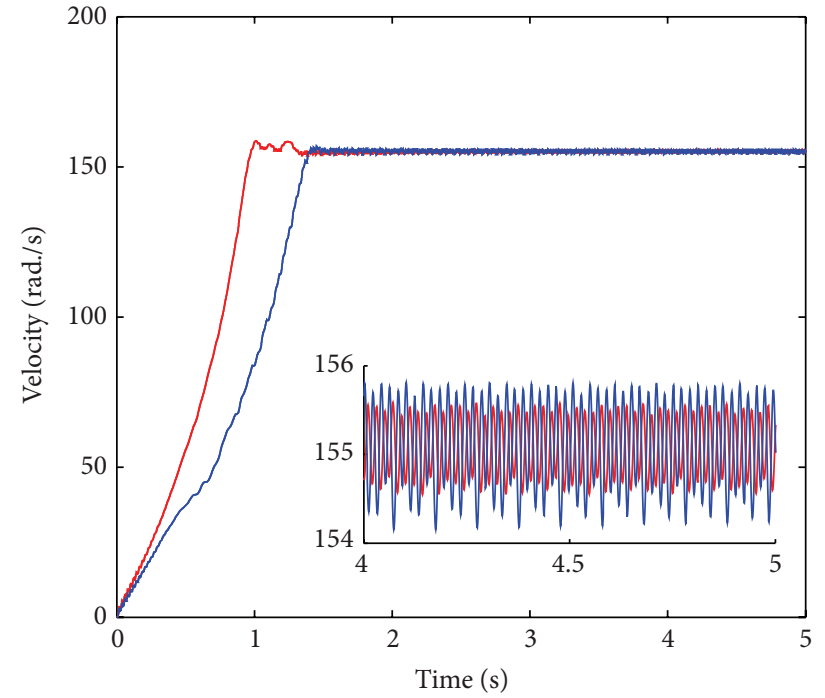

- Rotor 1

- Rotor 2

(a)

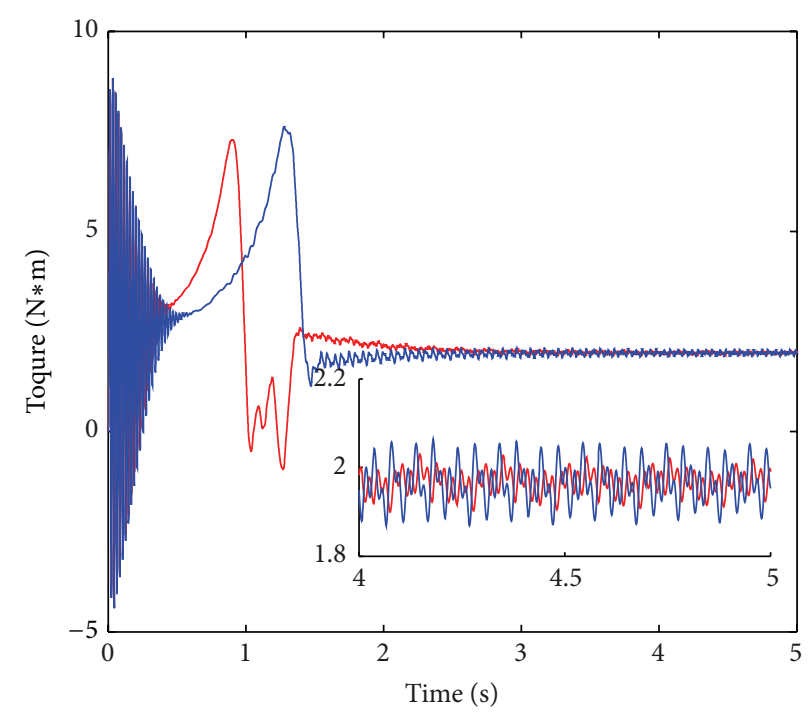

- Rotor 1

— Rotor 2

(c)

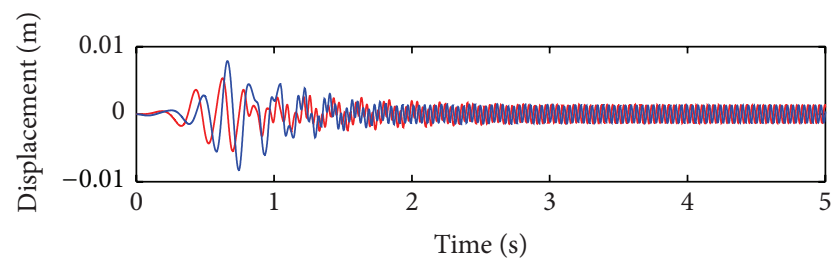

$-x$

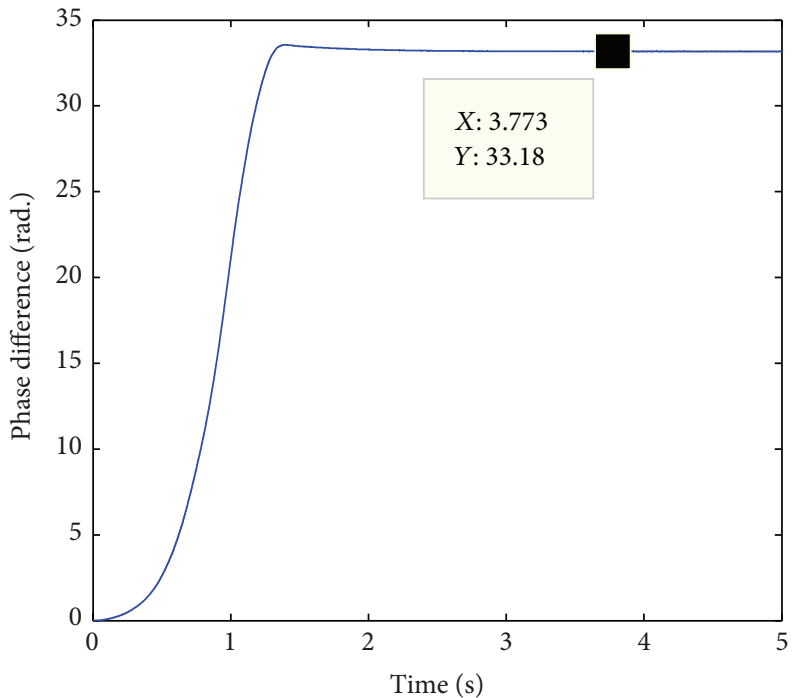

(b)

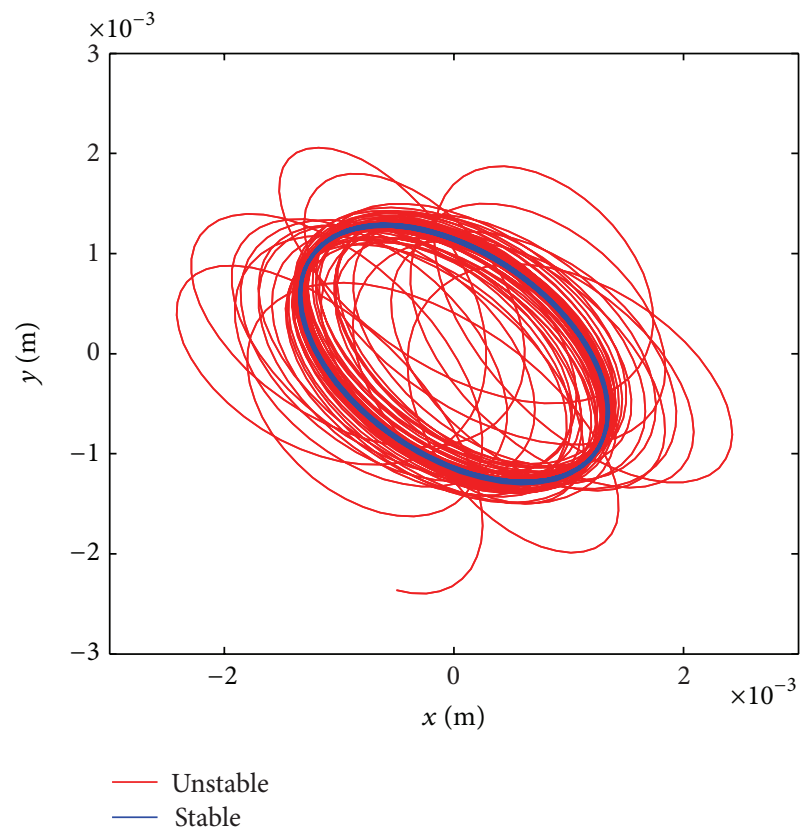

(d)

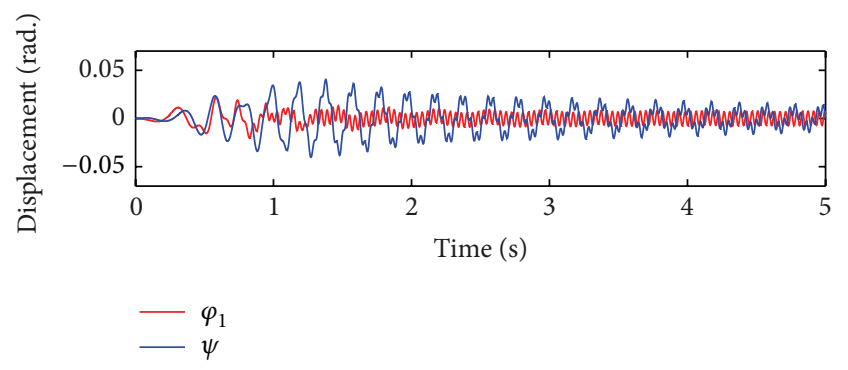

(f)

Figure 10: Simulation results for SACWA: (a) rotational velocity, (b) phase difference of the two rotors, (c) torques of the two rotors, (d) trajectory of mass center of the vibrobody, (e) displacement responses of the vibrobody in $x$ - and $y$-directions, and (f) displacement responses of the vibrobody in $\varphi_{1}$ - and $\psi$-directions. 


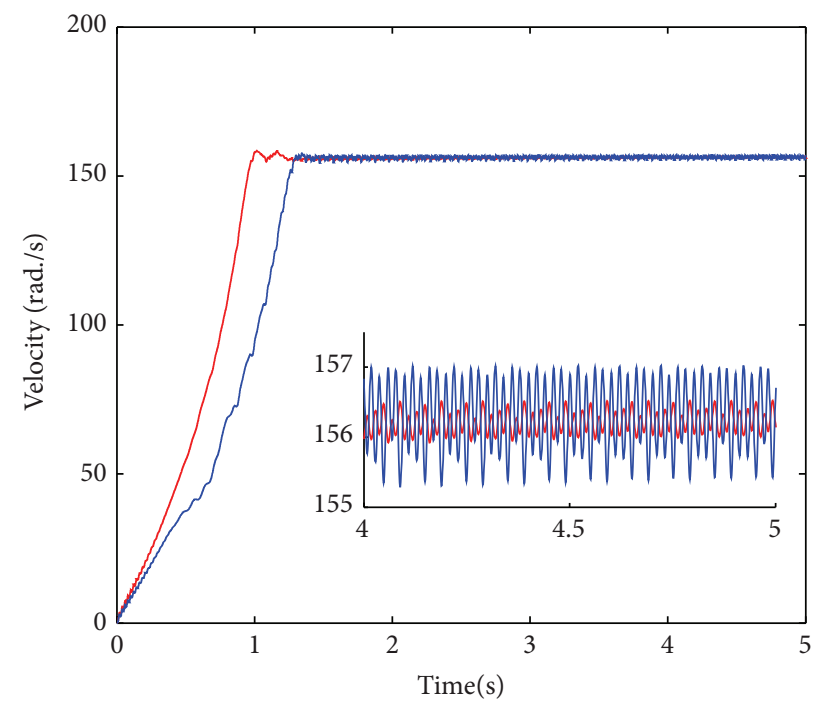

- Rotor 1

- Rotor 2

(a)

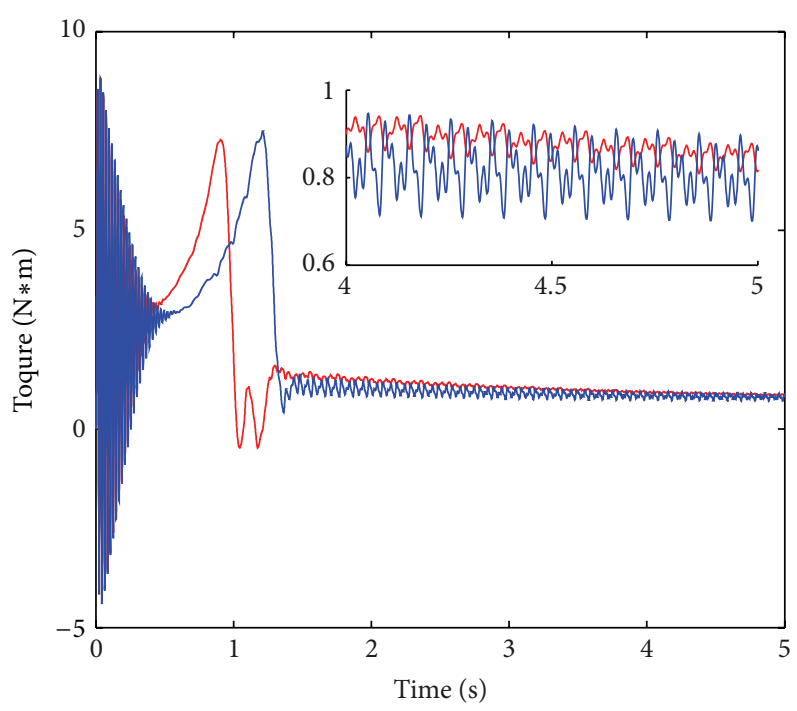

- Rotor 1

- Rotor 2

(c)

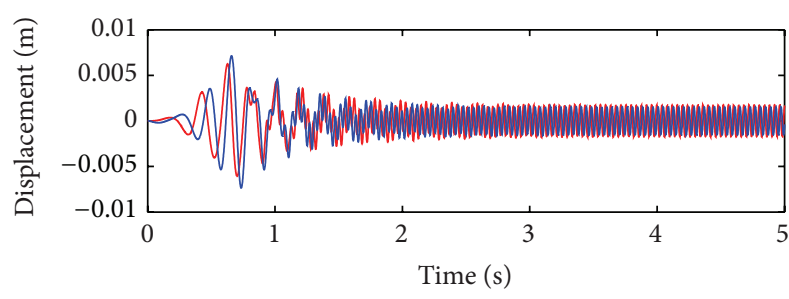

$-x$

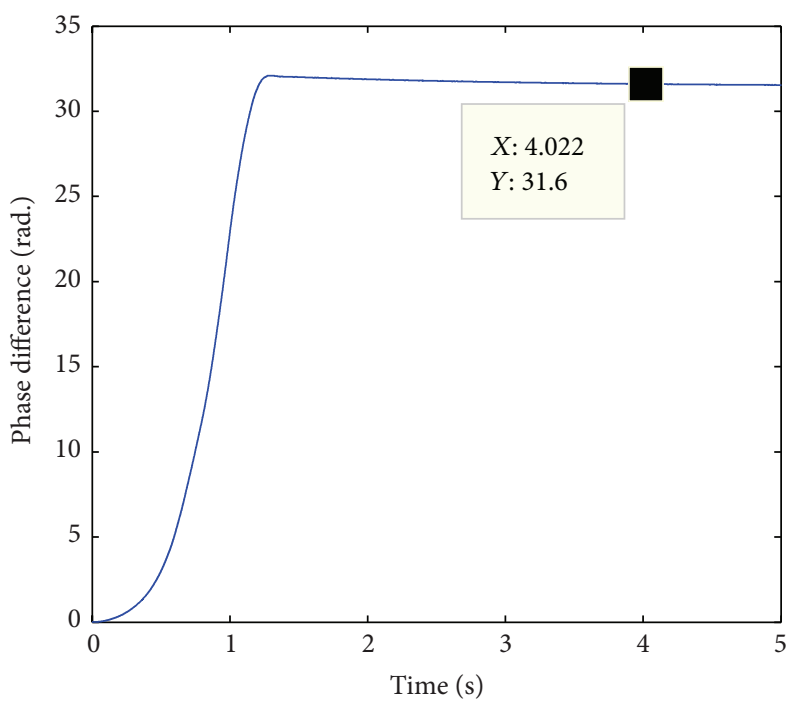

(b)

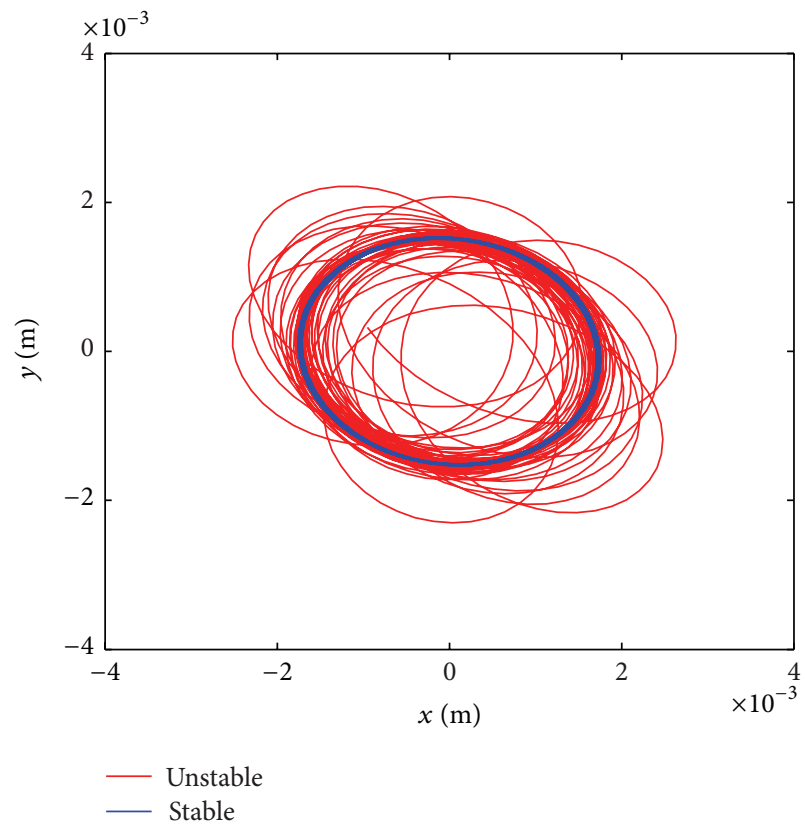

(d)

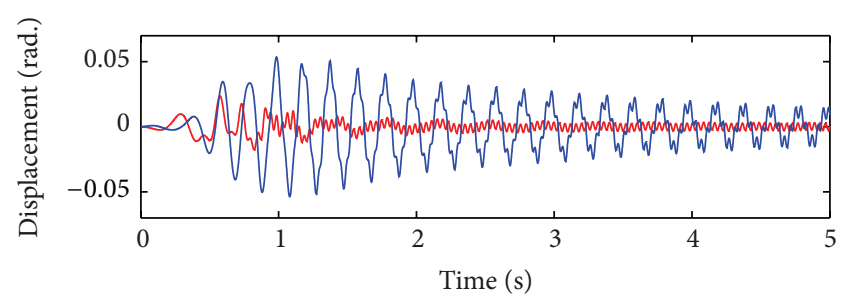

$\varphi_{1}$
$-\psi$

(e)

(f)

FIGURE 11: Simulation results for asymmetry rotors system: (a) rotational velocity, (b) phase difference of the two rotors, (c) torques of the two rotors, (d) trajectory of mass center of the vibrobody, (e) displacement responses of the vibrobody in $x$ - and $y$-directions, and (f) displacement responses of the vibrobody in $\varphi_{1}$ - and $\psi$-directions. 
the centrifugal forces produced by the two rotors are partially offset, so the amplitudes of the responses of the vibrobody are larger than that of Figure 9 (see Figure 10(e)). However, the stable trajectory of mass center of the vibrobody is an ellipse. Figure 10(f) represents the oscillating responds of the vibrobody in $\varphi_{1}$ - and $\psi$-directions. It can be seen that when the rotation velocity of the two rotors passes through the resonant region of the coupling system, the resonant responses of the vibrobody in the $x-, y-, \varphi_{1}$, and $\psi$-direction appeared in the starting process. Comparing simulation results with Figure 6(c), it should be stressed that the value of the stable phase difference is in accordance with the results obtained for the case of theoretical solutions (i.e., the stable phase difference with the theoretical calculation in Figure 6(c) is equal to 1.728 [rad]; here the stable phase difference with the computer simulation is 1.78 [rad]).

5.2.3. Synchronization for Asymmetry Rotors. To verify the synchronization problem of the asymmetry rotors, we take into account that the mass of the rotor, connected with pendulum rod, is nonidentical to another rotor. This simulation is performed on the assumption that $\eta_{13}=5, \eta_{23}=0.3$, $n_{1}=5, n_{x} \approx n_{y} \approx n_{\psi}=5.0, r_{o}=2.0, l=0.6$, and $\beta=0$. Finally, the simulation results are shown in Figure 11, from which one can see that, during the staring few seconds, angular acceleration of rotor 1 is greater than rotor 2 . The reason is that the moment of inertia of rotor 1 is smaller than that of rotor 2. Meanwhile, the operation velocity of the rotors stabilizes at $156[\mathrm{rad} / \mathrm{s}]$ in the synchronous state, and the synchronous phase difference between the rotors is $0.018[\mathrm{rad}]$ (as $33.18[\mathrm{rad}]=(10 \pi+0.018)[\mathrm{rad}])$. In this case, the synchronous state of the system is synphase synchronization, and note that the value of the phase difference with computer simulation is in agreement with the date label in Figure $8(\mathrm{c})$. So to speak, the nonidentity of mass between the rotors is independent of the synchronous state. With regard to the displacement amplitude of the vibrobody, the centrifugal forces produced by the two rotors are mutually added. However, the stable trajectory of mass center of the vibrobody is still an ellipse, which is the largest amplitude, then Section 5.2.2 and the smallest Section 5.2.1; hence, the amplitude of the vibrobody in synphase synchronization is larger than antiphase synchronization.

\section{Conclusions}

The occurrence of synchronized motion in the rotorpendulum system via the multi-DOF vibration has been investigated. Firstly, the dynamics equation of the system, deduced by Lagrange equation, has been converted into the dimensionless equations. Then Laplace transformation has been employed to solve the steady responses of the system. Next, the balance equation and the stability criteria of synchronization of the system have been derived using Poincare method base on the small parameter. Only should the values of the structure parameters satisfy the balance equation and the stability criteria, the stable phase difference can be acquired. Finally, the value of the phase difference has been calculated with numerical method; moreover, computer simulations have been performed to verify the correctness of the theoretical computations.

The analysis has revealed that the synchronous state of the rotor-pendulum system is mainly sensitive to the values of the spring stiffness coefficient, the length, and the angular installation of the pendulum rod. However, the synchronous state of the system is independent of the mass ratio between the two rotors and vibrobody, besides the operation velocity of the motors. With regard to the displacement amplitude of the vibrobody, the amplitude of the vibrobody in synphase synchronization is larger than antiphase synchronization. Meanwhile, reasonable values of the structure parameter are in favour of the implementing elliptical motion with large amplitude for vibrobody. Therefore, the vibration system proposed in this paper can be applied to design new balanced elliptical vibrating screens, when their structure parameters satisfy the balance equation and the stability criterion of synchronization. In the early stage, for the developing and understanding the internal characteristics of the system, we only consider the rotors under the assumption of the rotation in the same direction. Will these results change if the contra rotation of the rotors in the pendulum-rotor system is taken into account? We believe that finding the answer to this question is the next step in challenging task of getting a complete understanding of synchronization in such system.

\section{Conflict of Interests}

The authors declare that there is no conflict of interests regarding the publication of this paper.

\section{Acknowledgment}

This study is supported by National Natural Science Foundation of China (Grant no. 51074132) and Key Project of Talent Engineering of Sichuan (Grant no. 2016RZ0059).

\section{References}

[1] I. I. Blekhman, Synchronization in Science and Technology, ASME Press, New York, NY, USA, 1988.

[2] A. Pikovsky, M. Rosenblum, and J. Kurths, Synchronization-A Universal Concept in Nonlinear Sciences, Cambridge University Press, 2001.

[3] A. Arenas, A. Díaz-Guilera, J. Kurths, Y. Moreno, and C. Zhou, "Synchronization in complex networks," Physics Reports, vol. 469, no. 3, pp. 93-153, 2008.

[4] H. Zhang, X.-Y. Wang, X.-H. Lin, and C.-X. Liu, "Stability and synchronization for discrete-time complex-valued neural networks with time-varying delays," PLoS ONE, vol. 9, no. 4, Article ID e93838, 2014.

[5] W.-J. Yuan and C. Zhou, "Interplay between structure and dynamics in adaptive complex networks: emergence and amplification of modularity by adaptive dynamics," Physical Review E, vol. 84, no. 1, Article ID 016116, 2011.

[6] J. Peña Ramirez, K. Aihara, R. H. B. Fey, and H. Nijmeijer, "Further understanding of Huygens' coupled clocks: the effect 
of stiffness," Physica D: Nonlinear Phenomena, vol. 270, pp. 1119, 2014.

[7] V. Jovanovic and S. Koshkin, "Synchronization of Huygens clocks and the Poincaré method," Journal of Sound and Vibration, vol. 331, no. 12, pp. 2887-2900, 2012.

[8] P. Koluda, P. Perlikowski, K. Czolczynski, and T. Kapitaniak, "Synchronization configurations of two coupled double pendula," Communications in Nonlinear Science and Numerical Simulation, vol. 19, no. 4, pp. 977-990, 2014.

[9] R. Dilão, "Anti-phase synchronization and ergodicity in arrays of oscillators coupled by an elastic force," The European Physical Journal: Special Topics, vol. 223, no. 4, pp. 665-676, 2014.

[10] L. Marcheggiani, R. Chacón, and S. Lenci, "On the synchronization of chains of nonlinear pendula connected by linear springs," The European Physical Journal: Special Topics, vol. 223, no. 4, pp. 729-756, 2014.

[11] B. C. Wen, J. Fan, and C. Y. Zhao, Synchronization and Controled Sychronization in Engineering, Science Press, Beijing, China, 2009.

[12] L.-P. Zhang, H.-B. Jiang, and Q.-S. Bi, "Reliable impulsive synchronization for a class of nonlinear chaotic systems," Chinese Physics B, vol. 19, no. 1, Article ID 010507, 2010.

[13] X. Zhang, B. Wen, and C. Zhao, "Vibratory synchronization and coupling dynamic characteristics of multiple unbalanced rotors on a mass-spring rigid base," International Journal of Non-Linear Mechanics, vol. 60, pp. 1-8, 2014.

[14] P. Fang, Y. Hou, Y. Nan, and Z. Wang, "Synchronization of two homodromy rotors installed on a double vibro-body in a coupling vibration system," PLoS ONE, vol. 10, no. 5, Article ID e0126069, 2015.

[15] L. Sperling, B. Ryzhik, C. Linz, and H. Duckstein, "Simulation of two-plane automatic balancing of a rigid rotor," Mathematics and Computers in Simulation, vol. 58, no. 4-6, pp. 351-365, 2002.

[16] J. M. Balthazar, J. L. P. Felix, and R. M. L. R. F. Brasil, "Short comments on self-synchronization of two non-ideal sources supported by a flexible portal frame structure," Journal of Vibration and Control, vol. 10, no. 12, pp. 1739-1748, 2004.

[17] J. M. Balthazar, J. L. P. Felix, and R. M. Brasil, "Some comments on the numerical simulation of self-synchronization of four non-ideal exciters," Applied Mathematics and Computation, vol. 164, no. 2, pp. 615-625, 2005.

[18] A. A. N. Djanan, B. R. N. Nbendjo, and P. Woafo, "Effect of selfsynchronization of DC motors on the amplitude of vibration of a rectangular plate," European Physical Journal: Special Topics, vol. 223, no. 4, pp. 813-825, 2014.

[19] P. Fang, Q. Yang, Y. Hou, and Y. Chen, "Theoretical study on self-synchronization of two homodromy rotors coupled with a pendulum rod in a far-resonant vibrating system," Journal of Vibroengineering, vol. 16, no. 5, pp. 2188-2204, 2014. 


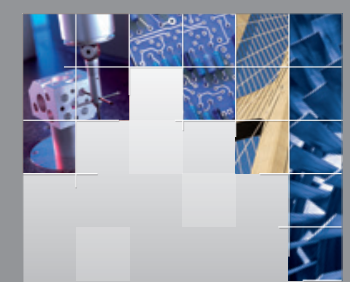

\section{Enfincering}
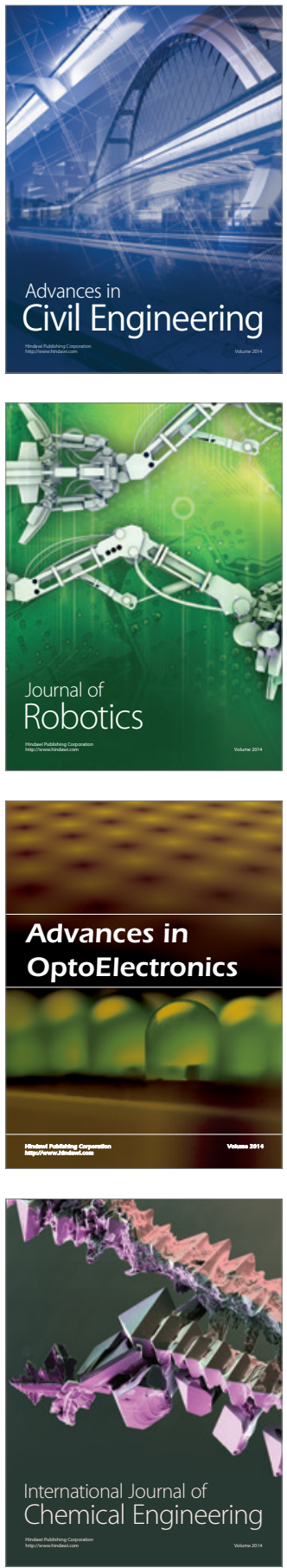

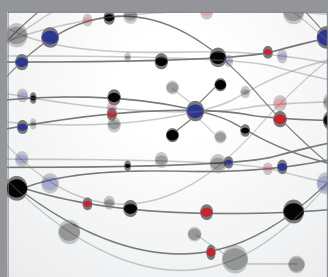

The Scientific World Journal

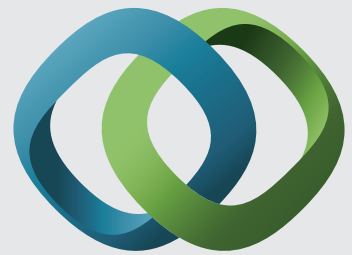

\section{Hindawi}

Submit your manuscripts at

http://www.hindawi.com
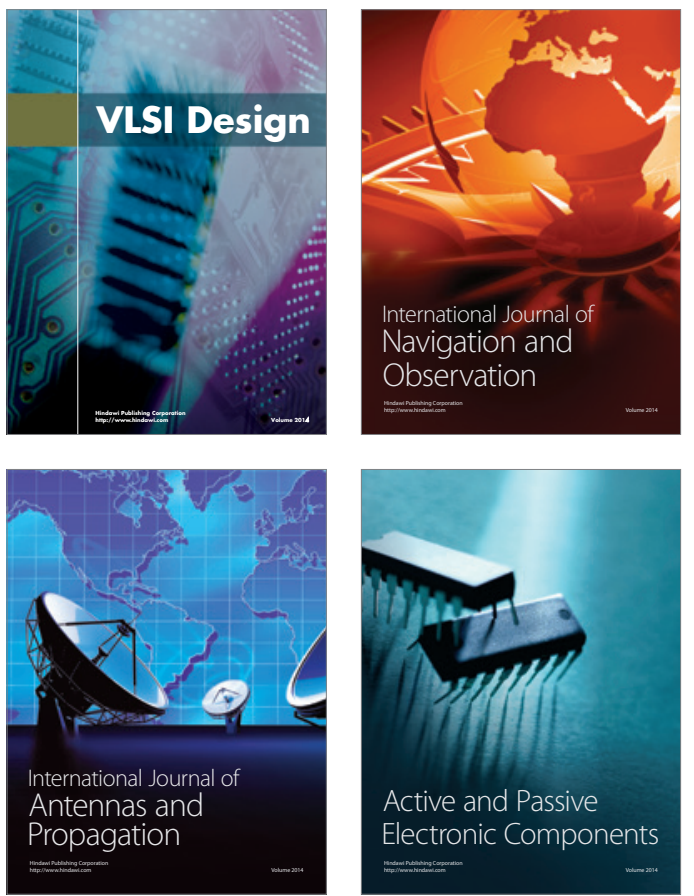
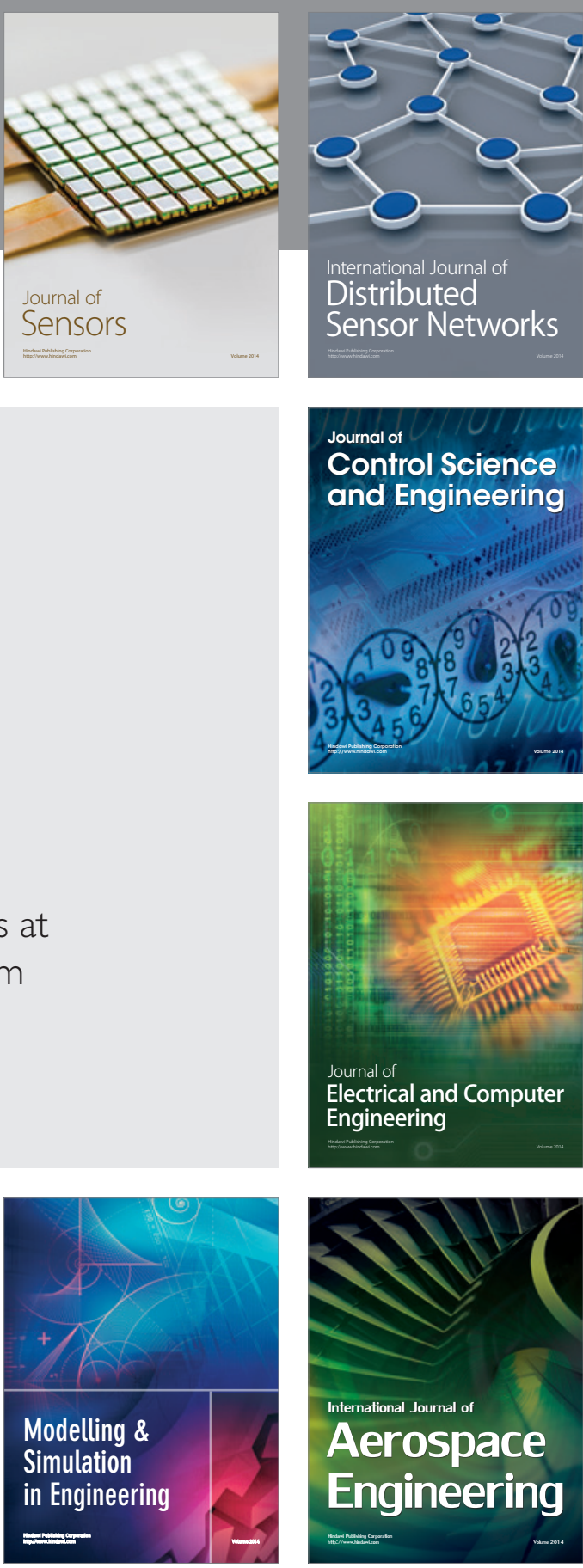

International Journal of

Distributed

Sensor Networks

Journal of

Control Science

and Engineering
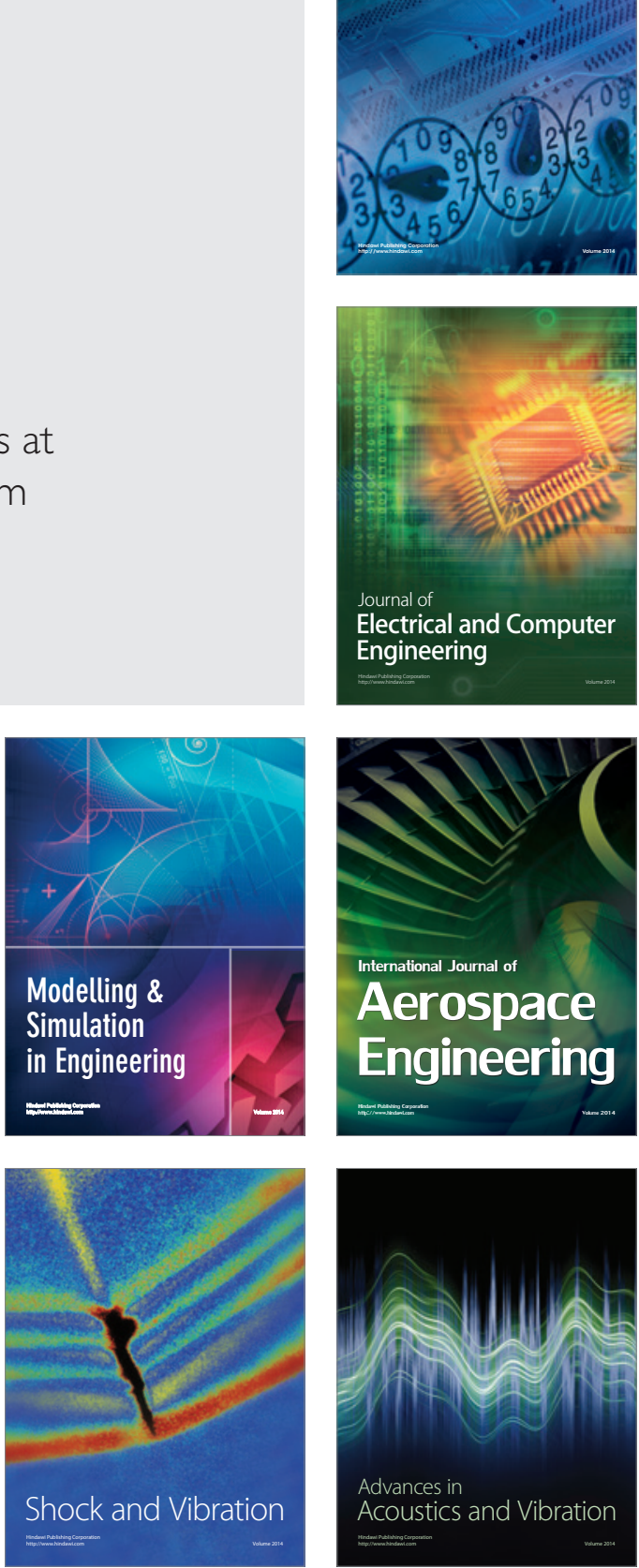